\title{
ON MECHANICAL QUADRATURES, IN PARTICULAR, WITH POSITIVE COEFFICIENTS*
}

BY

\author{
J. SHOHAT
}

\section{INTRODUCTION}

The present paper is an extension in various directions of a paper by Fejér. $†$

Consider a system of orthonormal polynomials $(O P)$

$$
\begin{aligned}
\phi_{n}(x ; a, b ; d \psi) & \equiv \phi_{n}(x ; d \psi) \equiv \phi_{n}(x)=a_{n}\left(x^{n}-S_{n-1} x^{n-1}+\cdots\right) \\
& \equiv a_{n} \Phi_{n}(x) ; \quad n=0,1,2, \cdots ; a_{n} \equiv a_{n}(d \psi)>0, \ddagger
\end{aligned}
$$

with the orthogonality property

$$
\int_{a}^{b} \phi_{m}(x) \phi_{n}(x) d \psi=\delta_{m n}=\left\{\begin{array}{ll}
0, & m \neq n ; \\
1, & m=n ;
\end{array} \quad m, n=0,1, \cdots .\right.
$$

Here and hereafter $\psi(x)$ denotes a bounded non-decreasing function in $(a, b)$, with infinitely many points of increase, including the end-points $a, b$. The limits $a$ and $b$ may be finite or infinite, but such that all moments

$$
\alpha_{n}=\int_{a}^{b} x^{n} d \psi, \quad n=0,1, \cdots, \text { with } \alpha_{0}>0
$$

exist. From (2) it follows that

$$
\int_{a}^{b} \Phi_{n}(x) G_{n-1}(x) d \psi=0, \quad \int_{a}^{b} \Phi_{n}(x) G_{n}(x) d \psi=\frac{g_{n}}{a_{n}^{2}}, \quad n=0,1, \cdots .
$$

Here $G_{-1}(x) \equiv 0$ and $G_{s}(x) \equiv \sum_{i=0}^{s} g_{i} x^{i}$ is a generic notation for an arbitrary polynomial of degree $\leqq s$, subject, in some instances, to certain explicitly stated conditions. The polynomials $\Phi_{n}(x) \equiv \Phi_{n}(x ; d \psi) \equiv \Phi_{n}(x ; a, b ; d \psi)$ satisfy the recurrence relation

* Presented to the Society, October 29, 1935; received by the editors January 29, 1936 and, in revised form, January 29, 1937.

$\dagger$ L. Fejér, Mechanische Quadraturen mit positiven Cotesschen Zahlen, Mathematische Zeitschrift, vol. 37 (1933), pp. 287-309.

¥ The notations employed are those of my monograph, Théorie générale des polynomes orthogonaux de Tchebycheff (hereafter referred to as M), Mémorial des Sciences Mathématiques, fasc. 66 (1934), to which the reader is referred for further details. 


$$
\begin{aligned}
& \Phi_{n}(x)=\left(x-c_{n}\right) \Phi_{n-1}(x)-\lambda_{n} \Phi_{n-2}(x), \quad n \geqq 2, \\
& \left(\Phi_{0}(x)=1, \Phi_{1}(x)=x-c_{1}\right), \\
& \lambda_{n}=\frac{a_{n}^{2}-2}{a_{n}^{2}-1}>0, \quad c_{n}=S_{n}-S_{n-1} .
\end{aligned}
$$

The zeros of $\Phi_{n}(x)$ are known to be real and distinct; they lie in $(a, b)$ and will be denoted by

$$
x_{i, n}(d \psi) \equiv x_{i, n} \equiv x_{i}, \quad \text { with } a<x_{1}<x_{2}<\cdots<x_{n}<b .
$$

Using the points (6) as abscissas in the Lagrange interpolation formula $(L I F)$ for a given function $f(x)$ which is finite at every point of $[a, b]$ and for which $\int_{a}^{b} f(x) d \psi$ exists, ${ }^{*}$ we are led to a Gaussian formula of mechanical quadratures (GMQ formula)

$$
\int_{a}^{b} f(x) d \psi \approx \sum_{i=1}^{n} H_{i, n} f\left(x_{i, n}\right), \quad H_{i, n}=\int_{a}^{b} \frac{\phi_{n}(x) d \psi}{\left(x-x_{i, n}\right) \phi_{n}{ }^{\prime}\left(x_{i, n}\right)},
$$

with the following properties:

All "coefficients" $H_{i, n}$ are positive, namely,

$$
H_{i, n} \equiv H_{i, n}(d \psi)=\int_{a}^{b}\left[\frac{\phi_{n}(x)}{\left(x-x_{i, n}\right) \phi_{n}^{\prime}\left(x_{i, n}\right)}\right]^{2} d \psi, \quad i=1,2, \cdots, n ;
$$

Formula (7) is exact for any polynomial of degree $\leqq 2 n-1$, i.e.,

$$
\int_{a}^{b} f(x) d \psi=\sum_{i=1}^{n} H_{i, n} f\left(x_{i, n}\right)+R_{n}(f), \quad \text { with } \quad R_{n}\left(G_{2 n-1}\right)=0 .
$$

The property (8.1) is of importance in connection with the convergence properties of (7), as shown by Fejér (loc. cit.) and as will be developed below. The question then naturally arises: does there exist, besides (6), some other choice of the points $x_{i, n}$ which yields a mechanical quadratures formula (MQF) with positive coefficients? Fejér's answer is in the affirmative in the case where $(a, b)$ is finite, $d \psi \equiv d x$, and the abscissas $x_{i, n}$ are the zeros of the polynomial

$$
P_{n}(x)+A P_{n-1}(x)+B P_{n-2}(x),
$$

where $P_{n}(x)$ is the Legendre polynomial of degree $n, A$ and $B$ are real constants, with $B \leqq 0$, provided the zeros in question are real and distinct and belong to the (closed) interval $[a, b] . \dagger$

\section{Stieltjes.}

* Throughout this paper integrals like $\int_{a}^{b} f(x) d \psi$ are understood to be taken in the sense of

$\dagger$ The various constants dealt with in this paper are assumed to be real, unless explicitly stated to the contrary. 
The object of the present paper is to give a more general answer to the foregoing question. We may mention the following direct generalization of Fejér's result. The polynomial $\Phi_{n}(x)+A \Phi_{n-1}(x)+B \Phi_{n-2}(x)$, where $\left\{\Phi_{n}(x)\right\}$ is any sequence of $O P$ and the constants $A, B$ are arbitrarily chosen, subject only to the limitation $B \leqq 0$, has all zeros real and distinct. Employing these zeros as abscissas, we get $M Q F(7)$, with all coefficients positive. Moreover, if $(a, b)$ is finite the $M Q F$ in question converges, i.e., $\lim _{n \rightarrow \infty} R_{n}(f)=0$, for any bounded $f(x)$ for which $\int_{a}^{b} f(x) d \psi$ exists, regardless of the distribution of abscissas relative to $(a, b)$. We obtain similar results concerning the more general polynomial

$$
\omega_{n}(x)=\Phi_{n}(x)+A_{1} \Phi_{n-1}(x)+\cdots+A_{k-1} \Phi_{n-k+1}(x) .
$$

Various properties of $O P$ are essential for our discussion. The most important point is to show that under sufficiently general conditions the terms in our $M Q F$ corresponding to abscissas outside $(a, b)$ do not affect the convergence properties for polynomials.

In connection with our main objective we make a general study of the zeros of $\omega_{n}(x)$. We also link the $M Q$ formulae under consideration with the theory of algebraic continued fractions, and we show that the $M Q$ formulae related to $O P$ are a powerful tool in the general study of such polynomials. At the outset we give some properties of the coefficients and the abscissas of any $M Q F$ based on $L I F$, extending and generalizing results due to Stekloff. $\dagger$

\section{MEChanical QUADRATURES FORMUlaE BASED ON LAGRANGE INTERPOLATION}

1. Construction. Let $f(x)$ be single-valued and finite at every point of a certain interval $(a, b)$ (closed, if it is finite). Choose $n$ distinct points

$$
c_{1}<c_{2}<\cdots<c_{n}
$$

and construct the associated $L I F$

$$
\begin{aligned}
f(x) & \approx \sum_{i=1}^{n} \frac{\omega_{n}(x)}{\left(x-c_{i}\right) \omega_{n}^{\prime}\left(c_{i}\right)} f\left(c_{i}\right), \quad f(x)=\sum_{i=1}^{n} \frac{\omega_{n}(x)}{\left(x-c_{i}\right) \omega_{n}^{\prime}\left(c_{i}\right)}+\rho_{n}(f), \\
\omega_{n}(x) & =\left(x-c_{1}\right)\left(x-c_{2}\right) \cdots\left(x-c_{n}\right), \quad \rho_{n}\left(G_{n-1}\right)=0 .
\end{aligned}
$$

By integration, we obtain from (10) a Lagrangian $M Q F$ ( $L M Q$ formula)

$\dagger \mathrm{W}$. Stekloff, Sur le problème de représentation des fonctions a l'aide de polynomes, du calcul approché des intégrales définies, du développment de fonctions en séries infinies suivant les polynomes et de l' interpolation, considérés au point de vue de Tchebycheff. Proceedings of the International Mathematical Congress, Toronto, 1924, vol. I, pp. 631-640. 
which may be spoken of as "generated" by the polynomial $\omega_{n}(x) .{ }^{*}$ Assuming the existence of $\int_{a}^{b} f(x) d x$, we have

$$
\begin{aligned}
& \int_{a}^{b} f(x) d \psi \equiv \sum_{i=1}^{n} C_{i} f\left(c_{i}\right), \quad C_{i}=\int_{a}^{b} \frac{\omega_{n}(x) d \psi}{\left(x-c_{i}\right) \omega_{n}^{\prime}\left(c_{i}\right)}, \\
& \int_{a}^{b} f(x) d \psi=Q_{n}(f)+R_{n}(f), \quad Q_{n}(f) \equiv \sum_{i=1}^{n} C_{i} f\left(c_{i}\right) .
\end{aligned}
$$

In particular

$$
R_{n}\left(G_{n-1}\right)=0, \quad \text { so that } \quad \alpha_{0}=\int_{a}^{b} d \psi=\sum_{i=1}^{n} C_{i} .
$$

We call the points $c_{i}$ "abscissas," the coefficients $C_{i}$ "coefficients" (Cotes' numbers) of the $M Q F$ under consideration.

In dealing with sequences of $M Q$ formulae corresponding to $n=N$, $N+1, \cdots$, we shall use the notations

$$
c_{i, n}, \quad C_{i, n}, \quad i=1,2, \cdots, n .
$$

The following remark is important. If some of the abscissas fall outside the closed interval $[a, b], \dagger$ we may assign arbitrary finite values to the corresponding $f\left(c_{i}\right)$ in (11), for this evidently does not affect the value of $\int_{a}^{b} f(x) d \psi$, but only that of $R_{n}(f)$ which, by definition, represents the difference $\int_{a}^{b} f(x) d \psi-Q_{n}(f)$. We agree, in case $f(x)$ is not a polynomial, to let $f\left(c_{i}\right)=0$, if $c_{i}$ is outside $[a, b]$. In other words, if $f(x)$ is not a polynomial, the summation $Q_{n}(f) \equiv \sum_{i=1}^{n} C_{i} f\left(c_{i}\right)$ is extended over only such $c_{i}$ as belong to $[a, b]$. When dealing with polynomials we keep all terms in the above summation in order not to destroy the important property $R_{n}\left(G_{n-1}\right)=0$.

2. Degree of precision of $L M Q$ formulae. If the abscissas in (11) are so chosen that

$$
R_{n}\left(G_{q}\right)=0
$$

but for one, at least, $G_{q+1}(x), R_{n}\left(G_{q+1}\right) \neq 0$, then $q$ is called "degree of precision" of our $L M Q$ formula $\ddagger$ (Stekloff, loc. cit.). Here $q$ may have any value from $n-1$, which corresponds to a random choice of abscissas, to $2 n-1$

* P6lya (Ueber die Konvergenz von Quadraturverfahren, Mathematische Zeitschrift, vol. 37 (1933), pp. 264-286) discusses $M Q F \int_{a}^{b} f(x) d x=\sum_{i=1}^{n} \lambda_{i} f\left(c_{i}\right)$, where the coefficients $\lambda_{i}$ are chosen according to a certain fixed rule, not necessarily connected with interpolation.

† If $c_{i} \leqq a$ (or $\left.c_{i} \geqq b\right)$, it will be assumed that $a$ (or $b$ ) is finite.

$\ddagger$ The expression "MQ formula" means here and hereafter a formula with $n$ abscissas, where $n$ is fixed, unless specified otherwise. 
in $G M Q$ formula (7). $2 n-1$ is the highest possible degree of precision, for $R_{n}\left(G_{2 n}\right)=0$ leads to a contradiction, namely:

$$
\int_{a}^{b} \omega_{n}^{2}(x) d \psi=\sum_{i=1}^{n} C_{i} \omega_{n}^{2}\left(c_{i}\right)=0 .
$$

ThEOREM I. A necessary and sufficient condition that $q=2 n-k$ be the degree of precision of $L M Q$ formula (11) is that

$$
\int_{a}^{b} \omega_{n}(x) G_{q-n}(x) d \psi=0,
$$

which is equivalent to the statement that $\omega_{n}(x)$ admits of the following representation

$$
\omega_{n}(x)=\Phi_{n}(x)+A_{1} \Phi_{n-1}(x)+\cdots+A_{k-1} \Phi_{n-k+1}(x),{ }^{*}
$$

where the $A_{i}$ are arbitrary constants.

The first part is readily proved by integration, upon combining (4) with the relations

$$
G_{q}(x)=\omega_{n}(x) \tilde{x}_{q-n}(x)+G_{n-1}(x), \quad G_{q}\left(c_{i}\right)=\tilde{\pi}_{n-1}\left(c_{i}\right), \quad i=1,2, \cdots, n .
$$

The second part is obvious. Observing that

$$
G_{q+1}(x)=\omega_{n}(x) G_{q-n+1}(x)+G_{n-1}(x),
$$

we immediately obtain the following formula:

$$
\int_{a}^{b} G_{q+1}(x) d \psi=\frac{A_{k-1} g_{k+1}}{a_{n-k+1}^{2}}+\sum_{i=1}^{n} C_{i} G_{q+1}\left(c_{i}\right) .
$$

THEOREM II. The polynomial

$$
\omega_{n}(x)=\Phi_{n}(x)+A_{1} \Phi_{n-1}(x)+\cdots+A_{k-1} \Phi_{n-k+1}(x),
$$

satisfies the orthgonality relation (13), with $q=2 n-k$, and changes sign in $(a, b)$ at least $n-k+1$ times.

The first part is evident, while the second is easily proved by an argument well known in the theory of $O P$.

3. Signs of the coefficients; location of the abscissas. Denote by $C_{i}^{\prime}$ the positive among the coefficients $C_{i}$ in (11) and by $C_{i}^{\prime \prime}$ the remaining ones (negative or vanishing). Let $c_{i}^{\prime}, c_{i}^{\prime \prime}$ be the corresponding abscissas, and $P$ and $N$ be the number of $C_{i}^{\prime}$ and $C_{i}^{\prime}$ respectively.

THEOREM III. If $q$ is the degree of precision of LMQ formula (11), then $n \geqq P \geqq[(q+2) / 2]$, so that $0 \leqq N \leqq[(2 n-q-1) / 2]$.

* By virtue of the recurrence relation (5), a polynomial of the form $\Phi_{n}(x)+P_{1}(x) \Phi_{n-1}(x)+\cdots$ $+P_{\nu}(x) P_{n-\nu}(x)\left(P_{i}(x)\right.$ - polynomial of degree $\left.\leqq i\right)$ can be written in the form (14). 
This result is due to Stekloff (loc. cit.). It is readily established by applying our $M Q F$ to the polynomial $G_{(q-1) / 2}(x)$ if $q$ is odd, or to $G_{q / 2}(x)$ if $q$ is even. These polynomials vanish at all $c_{i}^{\prime}$.

Further indications as to the location of the $c_{i}$ and more particularly, of the $c_{i}^{\prime}$ and $c_{i}^{\prime \prime}$, are given in the following theorems.

TheOREM IV. Let $D_{n}(x)$ be a polynomial of degree $n$ satisfying the orthogonality relation $\int_{a}^{b} D_{n}(x) G_{\nu}(x) d \psi=0$. If $\psi(x)$ is constant in $(\alpha, \beta) \subset(a, b)$, then $D_{n}(x)$ may change sign in $(\alpha, \beta)$ at most $n-\nu+\theta$ times, where $\theta=0$ or 1 , according as $n$ is odd or even. It follows that in an LMQ formula, with degree of precision $q$, at most $2 n-q+\theta$ abscissas may lie inside an interval of constancy of $\psi(x)$, where $\theta=0$ or 1 , according as $2 n-q$ is odd or even.

If $(\alpha, \beta)$ contains more than $n-\nu+\theta$ points where $D_{n}(x)$ changes sign, take any $\mu=n-\nu+\theta+1$ of these, say $\beta_{j+1}, \beta_{j+2}, \cdots, \beta_{j+\mu}$. We have, by hypothesis,

$$
\begin{aligned}
0 & =\int_{a}^{b} D_{n}(x) \frac{D_{n}(x) d \psi}{\left(x-\beta_{j+1}\right) \cdots\left(x-\beta_{j+\mu}\right)} \\
& =\int_{a}^{\alpha} \frac{D_{n}^{2}(x) d \psi}{\left(x-\beta_{j+1}\right) \cdots\left(x-\beta_{j_{\mu}}\right)}+\int_{\beta}^{b} \cdots,
\end{aligned}
$$

which is impossible, both integrals on the right being $>0$. This is a generalization of a known property of $O P(n-\nu=1)$, which is usually proved by means of Tchebycheff inequalities for the coefficients $H_{i, n}$ in (7), (see below, §11). The above proof (suggested by A. N. Milgram) is a simple application of the orthogonality property of $D_{n}(x)$.

THEOREM V. (i) If a certain $c_{j}^{\prime \prime}$ coincides with an end-point $a$ or $b$, then there exists $a c_{i}^{\prime}>a$ or $<b$ respectively. (ii) If $2 \nu$ denotes the greatest integer contained in $q-2 N-1$, then neither of the intervals $(-\infty, a],[b, \infty)$ may contain more than $P-\nu-1$ of the $c_{i}^{\prime} *^{*}$

To prove part (i), assume for definiteness $c_{j}^{\prime \prime}=a, n$ odd. By Theorem III, $N-1 \leqq(n-3) / 2$, and we may construct a $G_{(n-3) / 2}(x)$ such that $G_{(n-3) / 2}\left(c_{i}^{\prime \prime}\right)$ $=0$ at all $c_{i}^{\prime \prime}$, except for $c_{j}^{\prime \prime}=a$. Moreover, $q \geqq n-1>n-2$,

$$
0>\int_{a}^{b}(a-x) G_{(n-3) / 2}^{2}(x) d \psi=\sum C_{i}^{\prime}\left(a-c_{i}^{\prime}\right) G_{(n-3) / 2}^{2}\left(c_{i}^{\prime}\right),
$$

which shows that not all $c_{i}^{\prime}$ lie to the left of $a$.

To prove part (ii), note that $q \geqq 2 N+2 \nu+1$. Construct $G_{N+\nu}(x)$ such that $G_{N+\nu}(x)=0$ at all $c_{i}^{\prime \prime}$ and at any $\nu$ of the $c_{i}^{\prime}$, say, at $c_{i_{1}}{ }^{\prime}, c_{i_{2}}{ }^{\prime}, \cdots, c_{i_{\nu}}{ }^{\prime}$. Apply-

* We necessarily have $P-\nu-1 \geqq 0$, for $\nu \leqq(q-2 N-1) / 2 \leqq(2 n-2-2 N) / 2=n-N-1=P-1$. 
ing our $L M Q$ formula $(11)$ to $(x-a) G_{N+\nu}^{2}(x)$ and $(b-x) G_{N+\nu}^{2}(x)$ we obtain

$$
\begin{aligned}
& 0<\int_{a}^{b}(x-a) G_{N+\nu}^{2}(x) d \psi=\sum C_{i}^{\prime}\left(c_{i}^{\prime}-a\right) G_{N+\nu}^{2}\left(c_{i}^{\prime}\right), \\
& 0<\int_{a}^{b}(b-x) G_{N+\nu}^{2}(x) d \psi=\sum C_{i}^{\prime}\left(b-c_{i}^{\prime}\right) G_{N+\nu}^{2}\left(c_{i}^{\prime}\right), \quad c_{i}^{\prime} \neq c_{i_{1}}^{\prime}, \cdots, c_{i_{\nu}}^{\prime},
\end{aligned}
$$

which shows that the remaining $P-\nu$ of the $c_{i}^{\prime}$ cannot all lie in $(-\infty, a]$ or in $[b, \infty)$.

The above theorems readily yield the following corollaries.

COROLLARY 1. Every LMQ formula has at least $[(n+1) / 2]$ positive coeffcients.

COROLlaRY 2. If the polynomial (14) has all zeros real and distinct, the LMQ formula with degree of precision $q=2 n-k$, which uses these zeros as $a b$ scissas, has at least $[(2 n-k+2) / 2]$ positive coefficients, hence, at most, $[(k-1) / 2]$ negative or vanishing coefficients.

COROLlaRY 3. If all zeros of the polynomial (14) are real and distinct, and the associated LMQ formula has all coefficients positive, then neither of the intervals $(-\infty, a],[b, \infty)$ may contain more than $[k / 2]$ abscissas.

Some special cases. $(\alpha) k=1$. Here $q=2 n-1, n-k+1=n, P=n, N=0$, and we have a $G M Q$ formula.

( $\beta) k=2$, i.e.,

$$
\omega_{n}(x)=\Phi_{n}(x)+A_{1} \Phi_{n-1}(x), \quad A_{1} \neq 0 .
$$

Here $q=2 n-2, n-k+1=n-1,[k / 2]=1,[(k-1) / 2]=0, P=n, N=0$; all zeros are real and simple, with one, at most, $\leqq a$ or $\geqq b$, and all coefficients are positive.

$(\gamma) k=3$, i.e.,

$$
\omega_{n}(x)=\Phi_{n}(x)+A_{1} \Phi_{n-1}(x)+A_{2} \Phi_{n-2}(x), \quad A_{2} \neq 0 .
$$

Here at least $n-2$ zeros are real and lie between $a$ and $b$. Assuming further that all zeros are real and simple, we get for the corresponding $L M Q$ formula

$$
q=2 n-3, \quad P \geqq n-1, \quad N \leqq 1,
$$

so that one, at most, of the coefficients $C_{i}$ may become negative or vanish.

( $\delta) k=4$, i.e.,

$$
\omega_{n}(x)=\Phi_{n}(x)+A_{1} \Phi_{n-1}(x)+A_{2} \Phi_{n-2}(x)+A_{3} \Phi_{n-3}(x), \quad A_{3} \neq 0 .
$$


Here at least $n-3$ zeros are real and lie between $a$ and $b$. If all zeros are real and simple, then for the corresponding $L M Q$ formula

$$
q=2 n-4, \quad P \geqq n-1, \quad N \leqq 1 .
$$

By virtue of Theorem $\mathrm{V}$, we find that in both cases $(\gamma)$ and $(\delta)$ neither interval $(-\infty, a],[b, \infty)$ may contain more than two $c_{i}^{\prime}$, and not more than one $c_{i}^{\prime}$ if $N=0$.

4. Second method for studying the coefficients and abscissas. We now proceed to study the $c_{i}, C_{i}$ by another method which tells us more about the coefficients if we know more about the abscissas. Thus, the results previously obtained will be supplemented and extended.

Consider again an $L M Q$ formula with degree of precision $q=2 n-k$. We change the previous notations and divide the abscissas into two groups as follows.

$$
\begin{aligned}
& s=s_{1}+s_{2} \text { exterior abscissas : } \\
& a^{(1)}<a^{(2)}<\cdots<a^{\left(s_{1}\right)} \leqq a, \quad b^{\left(s_{2}\right)}>b^{\left(s_{2}-1\right)}>\cdots>b^{(1)} \geqq b, \\
& \nu=n-s \text { interior abscissas : } \\
& \quad a<c^{(1)}<c^{(2)}<\cdots<c^{(\nu)}<b,
\end{aligned}
$$

with the corresponding coefficients

$$
C_{a^{(i)}} \equiv C^{(i)}, \quad C_{b^{(j)}} \equiv C^{(j)}, \quad C_{c^{(l)}} \equiv C^{(l)},
$$

so that

$$
\begin{aligned}
\omega_{n}(x) & =(-1)^{s_{2}} \Pi(x) \Phi(x), \\
\Pi(x) & =\prod_{i=1}^{s_{1}}\left(x-a^{(i)}\right) \prod_{j=1}^{s_{2}}\left(b^{(j)}-x\right) \equiv \prod_{a}(x) \prod_{b}(x), \\
\Phi(x) & =\prod_{l=1}^{\nu}\left(x-c^{(l)}\right) .
\end{aligned}
$$

Note that $\Pi(x) \geqq 0$ in $[a, b]$ and $>0$ in $(a, b)$. We agree to replace $\Pi_{a}(x)$ or $\prod_{b}(x)$ by unity in case $s_{1}=0$ or $s_{2}=0$. Introduce a new system of $O P$ :

$$
\Phi_{n}\left(x ; d \psi_{1}\right) \equiv \Phi_{n}\left(x ; a, b ; d \psi_{1}\right), \quad d \psi_{1}(x)=\prod(x) d \psi(x) .^{*}
$$

Equation (13) can be rewritten as

$$
\int_{a}^{b} \Phi(x) G_{q-n}(x) d \psi_{1}=0 .
$$

* We recall that $\Phi_{n}(x)$ stands for the $O P$ with the highest coefficient unity, and $\phi_{n}(x)$ stands for the normalized $O P$ (see Introduction). 
We get at once the previous result:

$$
q-n=n-k<\nu=n-s, \quad \text { i.e., } \quad s<2 n-q=k
$$

(for otherwise we obtain a contradiction by taking in (22) $G_{q-n}(x) \equiv \Phi(x)$ ). Moreover, (22) leads to a new representation of $\omega_{n}(x)$ in terms of $\Phi_{n}\left(x ; d \psi_{1}\right)$ (supplementing (14)):

$$
\begin{array}{r}
\Phi(x)=\Phi_{\nu}\left(x ; d \psi_{1}\right)+B_{1} \Phi_{\nu-1}\left(x ; d \psi_{1}\right)+\cdots+ \\
\omega_{n}(x)=(-1)^{s_{2}} \prod(x)\left[\Phi_{\nu}\left(x ; d \psi_{1}\right)+B_{1} \Phi_{\nu-1}\left(x ; d \psi_{1}\right)+\cdots\right. \\
\left.+B_{k_{1}+1} \Phi_{\nu-k_{1}+1}\left(x ; d \psi_{1}\right)\right], \\
\left(B_{i}=\text { const., } \nu=n-s, k_{1}=k-s\right) .
\end{array}
$$

Upon rewriting (22) as

$$
\int_{a}^{b} \Phi(x) G_{q-8-(n-s)}(x) d \psi_{1}=0
$$

and recalling that $\Phi(x)$ is of degree $\nu=n-s$, we conclude that the interior abscissas in (19) originate a new LMQ formula, with $d \psi(x)$ replaced by $d \psi_{1}(x)=\prod(x) d \psi(x)$, namely:

$$
\int_{a}^{b} f(x) d \psi_{1} \approx \sum_{l=1}^{\nu} C_{l}^{\prime} f\left(c^{(l)}\right),
$$

with degree of precision $q_{1}=q-s=2 \nu-k_{1}, k_{1}=k-s$. Since $q_{1} \leqq 2(n-s)-1$, we see that

$$
q \leqq 2 n-s-1 \text {. }
$$

Hence, $2 n-s-1$ is the highest degree of precision attainable by the $L M Q$ formula (11) having $s$ preassigned exterior abscissas.

We proceed to establish relations between the coefficients of the two $L M Q$ formulae (11) and (24). Applying (11), where $\omega_{n}(x)$ is now replaced by $(-1)^{s_{2}} \prod(x) \Phi(x)$, we get:

$$
\begin{aligned}
& C^{(l)}=\frac{1}{\prod\left(c^{(l)}\right)} \int_{a}^{b} \frac{\Phi(x) d \psi_{1}}{\left(x-c^{(l)}\right) \Phi^{\prime}\left(c^{(l)}\right)}=\frac{C_{l}^{\prime}}{\prod\left(c^{(l)}\right)}, \quad l=1,2, \cdots, \nu, \\
& C^{(l)} \cdot C_{l}^{\prime}>0, \text { for all interior coefficients } C^{(l)} .
\end{aligned}
$$

Letting $s$ be fixed, consider some special cases.

(i) $q$ attains its highest value, i.e.,

$$
q=2 n-s-1, \quad k=s+1 .
$$

Here $\Phi(x)=\Phi_{\nu}\left(x ; d \psi_{1}\right), k_{1}=1, q_{1}=2 \nu-1$. Hence, (24) is a $G M Q$ formula, and

$$
C_{l}^{\prime}=H_{l, \nu}\left(d \psi_{1}\right), \quad l=1,2, \cdots, \nu .
$$


Furthermore, applying the original $L M Q$ formula (11) to

$$
G_{2 n-8-1}(x)=\prod_{a}(x) \prod_{b}(x) \Phi(x) \Phi_{\nu}\left(x ; d \psi_{1, i}\right) /\left(x-a^{(i)}\right), \quad d \psi_{1, i}=\frac{d \psi_{1}}{x-a^{(i)}},
$$

we get

$$
C^{(i)}=1 /\left\{a_{\nu}{ }^{2}\left(d \psi_{1, i}\right) \Phi\left(a^{(i)}\right) \Phi_{\nu}\left(a^{(i)} ; d \psi_{1, i}\right) \prod_{a}^{\prime}\left(a^{(i)}\right) \prod_{b}\left(a^{(i)}\right), i=1,2, \cdots, s_{1},\right.
$$

and similarly for the $C^{(j)}$. But (M, p. 27)

$$
\begin{aligned}
\Phi_{n}(\xi ; d \psi) \Phi_{n}(\xi ; d \bar{\psi}) & =K_{n}(\xi ; d \psi) / a_{n}^{2}(d \psi) \\
(\xi & \leqq a \text { or } \geqq b, d \bar{\psi}=|x-\xi| d \psi),
\end{aligned}
$$

where, by Darboux's formula,

(29) $K_{n}(x ; d \psi) \equiv K_{n}(x) \equiv \sum_{i=0}^{n} \phi_{i}{ }^{2}(x)=\frac{a_{n}}{a_{n+1}}\left[\phi_{n+1}{ }^{\prime}(x) \phi_{n}(x)-\phi_{n}{ }^{\prime}(x) \phi_{n+1}(x)\right]$.

We thus finally get the following formulae for the coefficients $C_{i}$ in (11):

Interior coefficients :

$$
C^{(l)}=\frac{H_{l, \nu}\left(d \psi_{1}\right)}{\Pi\left(c^{(l)}\right)}, \quad l=1,2, \cdots, \nu ;
$$

Exterior coefficients :

$$
\begin{gathered}
C^{(i)}=1 /\left\{\prod_{a}^{\prime}\left(a^{(i)}\right) \prod_{b}\left(a^{(i)}\right) K_{\nu}\left(a^{(i)} ; d \psi_{1, i}\right)\right\},{ }^{*} \\
C^{(j)}=1 /\left\{\prod_{a}\left(b^{(j)}\right) \prod_{b}^{\prime}\left(b^{(j)}\right) K_{\nu}\left(b^{(j)} ; d \psi_{1, j}\right)\right\} \\
\left(1 \leqq i \leqq s_{1} ; 1 \leqq j \leqq s_{2} ; d \psi_{1, i}=d \psi /\left(x-a^{(i)}\right), d \psi_{1, j}=d \psi /\left(b^{(j)}-x\right)\right) .
\end{gathered}
$$

This leads to

Theorem VI. Consider an LMQ formula with $s_{1}$ abscissas $a^{(1)}<a^{(2)}$ $<\cdots<a^{\left(s_{1}\right)} \leqq a$ and $s_{2}$ abscissas $b^{\left(s_{2}\right)}>b^{\left(s_{2}-1\right)}>\cdots>b^{(1)} \geqq b$, having the highest possible degree of precision $q=2 n-s-1=2 n-k$, with $s=s_{1}+s_{2}, k=s+1$. Then all interior coefficients are positive; the exterior coefficients alternate in sign, namely: $\operatorname{sgn} C^{(i)}=(-1)^{s_{1}-i}, \operatorname{sgn} C^{(j)}=(-1)^{j-1}\left(1 \leqq i \leqq s_{1}, 1 \leqq j \leqq s_{2}\right)$.

COROLLARY. If $q=2 n-k$, with $k=s_{1}+s_{2}+1$, then all the coefficients are positive if and only if $s_{1} \leqq 1, s_{2} \leqq 1$.

(ii) $q=2 n-s-2$, i.e., $q=2 n-k$, with $k=s+2$. Here again all interior coefficients are positive. In fact,

$$
k=2, \quad q_{1}=2 \nu-2, \quad \Phi(x)=\Phi_{\nu}\left(x ; d \psi_{1}\right)+B_{1} \Phi_{\nu-2}\left(x ; d \psi_{1}\right),
$$

* Cf. C. Winston, On mechanical quadratures formulae involving the classical orthogonal polynomials, Annals of Mathematics, vol. 35 (1934), pp. 658-677. 
so that, by the preceding discussion, all $C_{l}^{\prime}$, and hence, by (27), all $C^{(l)}$, are positive: We have

$$
C^{(l)} \Pi\left(c^{(l)}\right)=\int_{a}^{b} \Pi(x)\left\{\frac{\Phi(x)}{\left(x-c^{(l)}\right) \Phi^{\prime}\left(c^{(l)}\right)}\right\}^{2} d \psi, \quad l=1,2, \cdots, \nu .
$$

In particular, for $s=0$,

$$
C^{(l)} \equiv C_{i}=\int_{a}^{b}\left\{\frac{\omega_{n}(x)}{\left(x-c^{(l)}\right) \omega_{n}^{\prime}\left(c^{(l)}\right)}\right\}^{2} d \psi, \quad i=l=1,2, \cdots, \nu=n,
$$

so that for $q=2 n-1,2 n-2$, we have the same expression for $C_{i}$ (see (8.1)). Apply now (15) to the polynomials

$G_{2 n-s-1}(x)=\frac{\prod(x)}{x-a^{(i)}} \Phi^{2}(x), \frac{\prod(x)}{b^{(j)}-x} \Phi^{2}(x), \quad i=1,2, \cdots, s_{1} ; j=1,2, \cdots, s_{2}$.

Then

$$
\begin{aligned}
& (-1)^{s_{2}} A_{k-1} / a_{n-k+1}^{2}+C^{(i)}(-1)^{s_{1}-i}\left|\prod_{a}^{\prime}\left(a^{(i)}\right)\right| \Phi^{2}\left(a^{(i)}\right)>0, i=1,2, \cdots, s_{1}, \\
& (-1)^{s_{2}} A_{k-1} / a_{n-k+1}^{2}+C^{(j)}(-1)^{j}\left|\prod^{\prime}\left(b^{(j)}\right)\right| \Phi^{2}\left(b^{(j)}\right)<0, \quad j=1,2, \cdots, s_{2} .
\end{aligned}
$$

Hence, $(-1)^{s_{2}} A_{k-1}<0$ implies that the $C^{(i)}$ alternate in sign; $(-1)^{s_{2}} A_{k-1}>0$ implies that the $C^{(j)}$ alternate in sign.*

(iii) $q=2 n-k$, with $k=s+3, s+4$. Here we may have no more than one negative or vanishing coefficient, as is seen from

$$
\begin{array}{r}
0<\int_{a}^{b} \Pi(x)\left\{\frac{\Phi(x)}{\left(x-c^{\left(l_{1}\right)}\right)\left(x-c^{\left(l_{2}\right)}\right)}\right\}^{2} d \psi=h_{1} C^{\left(l_{1}\right)} \Pi\left(c^{\left(l_{1}\right)}\right)+h_{2} C^{\left(l_{2}\right)} \Pi\left(c^{\left(l_{2}\right)}\right), \\
1 \leqq l_{1}, \quad l_{2} \leqq \nu, \quad l_{1} \neq l_{2},
\end{array}
$$

where $h_{1}, h_{2}$ are certain positive constants. Similarly we prove the more general result: if $q=2 n-k$, with $k=s+r(r \geqq 1)$, then we may have no more than $[(r-1) / 2]$ negative or vanishing interior coefficients.

$L M Q$ formulae, where all coefficients are positive, enjoy special important properties as was indicated by Fejér (loc. cit.). It seems proper to call such formulae " $M Q$ formulae of Fejér's type" ( $F M Q$ formulae). The following section is devoted to their discussion.

* In particular, in case $q=2 n-4, s_{1}=s_{2}=1, A_{3}>0$ implies $C_{a}(1)>0$, and $A_{3}<0$ implies $C_{b}(1)>0$. If $C_{b}(1)<0$ or $C_{a}(1)<0$, then respectively:

$$
\begin{aligned}
& \left|C_{b}(1)\right|<\left|A_{3}\right| /\left\{a_{n}^{2}\left(d \psi_{1}\right)\left(b^{(1)}-a^{(1)}\right) \Phi^{2}\left(a^{(1)}\right)\right\}, \\
& \left|C_{a}(1)\right|<\left|A_{3}\right| /\left\{a_{n}^{2}{ }_{3}\left(d \psi_{1}\right)\left(b^{(1)}-a^{(1)}\right) \Phi^{2}\left(b^{(1)}\right)\right\} .
\end{aligned}
$$




\section{III. $F M Q$ FORMULAE}

5. Existence; properties of the abscissas. Using the previously introduced $s_{1}, s_{2}, s=s_{1}+s_{2}$, we state the following results.

( $\alpha) \omega_{n}(x)=\Phi_{n}(x)+A \Phi_{n-1}(x), A$ arbitrary, always generates an $F M Q$ formula ( $A=0$ yields a $G M Q$ formula).

( $\beta) \omega_{n}(x)=\Phi_{n}(x)+A_{1} \Phi_{n-1}(x)+A_{2} \Phi_{n-2}(x)\left(A_{2} \neq 0\right)$ generates an $F M Q$ formula, if $s_{1}=s_{2}=1$ or $s_{1}+s_{2}=1, A_{2}<0$. It cannot generate such a formula if $s_{1}=2$ or $s_{2}=2$ (here $s_{1}+s_{2} \leqq 2$ ).

$(\alpha)$ has been shown above; $(\beta)$ follows from the Corollary to Theorem VI and from (31), (32). It will be improved below.

The following result is important, since it deals with the $A_{i}$ only.

THEOREM VII. The polynomial

$$
\omega_{n}(x)=\Phi_{n}(x)+A_{1} \Phi_{n-1}(x)+A_{2} \Phi_{n-2}(x), \quad A_{2}<0,
$$

has, with any $\Phi_{n}(x ; a, b ; d \psi)$, all zeros real and distinct. It generates an FMQ formula.*

We already know that $n-2$ zeros are real and distinct and lie in $(a, b)$. It thus remains to prove that no double or imaginary zero may occur. This is readily achieved by means of Darboux's formula

$$
\begin{aligned}
K_{n}(x, y ; d \psi) & \equiv K_{n}(x, y) \equiv \sum_{i=0}^{n} \phi_{i}(x) \phi_{i}(y) \\
& =\frac{a_{n}}{a_{n+1}} \frac{\phi_{n+1}(x) \phi_{n}(y)-\phi_{n}(x) \phi_{n+1}(y)}{x-y} .
\end{aligned}
$$

Rewrite (33):

$$
a_{n} \omega_{n}(x)=\phi_{n}(x)+A_{1}^{\prime} \phi_{n-1}(x)+A_{2}^{\prime} \phi_{n-2}(x), \quad A_{2}^{\prime}<0 .
$$

Let $\omega_{n}(x)$ have a double zero $\xi$ or two conjugate imaginary zeros $\xi$, $\bar{\xi}$. In the first case

$\phi_{n}(\xi)+A_{1}^{\prime} \phi_{n-1}(\xi)+A_{2}^{\prime} \phi_{n-2}(\xi)=0, \quad \phi_{n}^{\prime}(\xi)+A_{1}^{\prime} \phi_{n-1}^{\prime}(\xi)+A_{2}^{\prime} \phi_{n-2}^{\prime}(\xi)=0$, whence, making use of (29),

$$
A_{2}^{\prime}=\frac{a_{n} a_{n-2}}{a_{n-1}^{2}} \cdot \frac{K_{n-1}(\xi)}{K_{n-2}(\xi)}>0 .
$$

In the second case we have

* Cf. Fejér (loc. cit., pp. 302-303), where the last part of this theorem is established for the case $\Phi_{n}(x) \equiv P_{n}(x)$-Legendre polynomial. 
$\phi_{n}(\xi)+A_{1}^{\prime} \phi_{n-1}(\xi)+A_{2}^{\prime} \phi_{n-2}(\xi)=0, \quad \phi_{n}(\bar{\xi})+A_{1}^{\prime} \phi_{n-1}(\bar{\xi})+A_{2}^{\prime} \phi_{n-2}(\bar{\xi})=0$, and we can use (34) with the result

$$
A_{2}^{\prime}=\frac{a_{n} a_{n-2}}{a_{n-1}^{2}} \cdot \frac{K_{n-1}(\xi, \bar{\xi})}{K_{n-2}(\xi, \bar{\xi})}>0 .
$$

The second part of Theorem VII is proved in precisely the same manner as in the special case considered by Fejér.

Corollary. For the polynomial (33) we can have neither $s_{1}=2$, nor $s_{2}=2$.

This follows from what was said in $(\beta)$ above.

The following simple example shows that if the condition $A_{2}<0$ is violated, the polynomial (33) may have a double zero. Take $a=-1, b=1$ and a "symmetric" sequence of $O P$, i.e., a sequence for which all $c_{n}$ in (5) vanish. The polynomial

$$
\omega_{2 n}(x)=\Phi_{2 n}(x)+\lambda_{2 n} \Phi_{2 n-2}(x) \equiv x \Phi_{2 n-1}(x)
$$

has a double zero at the origin.

Still more can be said about the abscissas in case $A_{2}=0$. We make use of the known property of $O P$ by which (see (6))

$$
a<x_{1, n}<x_{1, n-1}<x_{2, n}<x_{2, n-1}<\cdots<x_{n-1, n-1}<x_{n, n}<b,
$$

and state

THEOREM VIII. The zeros $c_{i, n}$ of the polynomial

$$
\omega_{n}(x)=\Phi_{n}(x)+A_{1} \Phi_{n-1}(x),
$$

where $A_{1}(\neq 0)$ is an arbitrary constant which may depend on $n$, are distributed as follows:

In case $A_{1}>0$,

$$
c_{1, n}<x_{1, n} ; x_{1, n-1}<c_{1, n-1}<x_{2, n} ; \cdots ; x_{n-1, n-1}<c_{n, n}<x_{n, n} .
$$

In case $A_{1}<0$,

$x_{1, n}<c_{1, n}<x_{1, n-1} ; x_{2, n}<c_{2, n}<x_{2, n-1} ; \cdots$;

$$
x_{n-1, n-1}<c_{n-1, n-1}<x_{n-1, n} ; x_{n, n}<c_{n, n} .
$$

The proof follows from the relations

$$
\begin{aligned}
\omega_{n}\left(x_{i, n}\right) \omega_{n}\left(x_{i+1, n}\right) & =A_{1}^{2} \Phi_{n-1}\left(x_{i+1, n}\right) \Phi_{n-1}\left(x_{i, n}\right)<0, \quad 1 \leqq i \leqq n-1, \\
\omega_{n}\left(x_{i, n-1}\right) \omega_{n}\left(x_{i+1, n-1}\right) & =\Phi_{n}\left(x_{i, n-1}\right) \Phi_{n}\left(x_{i+1, n-1}\right)<0, \quad 1 \leqq i \leqq n-2, \\
\operatorname{sgn}\left\{\omega_{n}\left(x_{1, n}\right) \omega_{n}\left(x_{1, n-1}\right)\right\} & =-\operatorname{sgn}\left\{\omega_{n}\left(x_{n, n}\right) \omega_{n}\left(x_{n-1, n-1}\right)\right\}=\operatorname{sgn} A_{1}, \\
\operatorname{sgn}\left\{\omega_{n}\left(x_{1, n}\right) \omega_{n}(-\infty)\right\} & =-\operatorname{sgn}\left\{\omega_{n}\left(x_{n, n}\right) \omega_{n}(+\infty)\right\}=-\operatorname{sgn} A_{1} .
\end{aligned}
$$


6. Upper bounds for the coefficients. The following theorem plays an important part in the study of the convergence properties of $F M Q$ formulae.

Theorem IX. Any coefficient $C_{i}(i=1,2, \cdots, n)$ of an FMQ formula, with degree of precision $q$, satisfies the inequality

$$
C_{i} \leqq \min \int_{a}^{b} G_{[q / 2]}^{2}(x) d \psi
$$

for all $G_{[q / 2]}(x)$ such that $G_{[q / 2]}\left(x_{i}\right)=1$. It follows (by known properties of $O P$ ) that

$$
C_{i} \leqq 1 / K_{[q / 2]}\left(c_{i} ; d \psi\right), \quad i=1,2, \cdots, n .
$$

In fact, for $G_{[q / 2]}(x)$ with the above property we have

$$
\int_{a}^{b} G_{[q / 2]}^{2}(x) d \psi=\sum_{l=1}^{n} C_{l} G_{[q / 2]}^{2}\left(c_{l}\right) \geqq C_{i} .
$$

7. The convergence properties. Hereafter, the interval $(a, b)$ is assumed to be finite. The case of an infinite interval will be treated elsewhere, but many of the results here obtained hold for an infinite interval as well.

We further assume to have given an infinite sequence of polynomials

$$
\begin{aligned}
\omega_{n} \equiv \omega_{n}(x)=\Phi_{n}(x)+A_{1} \Phi_{n-1}(x)+\cdots+A_{k-1} \Phi_{n-k+1}(x) \\
\left(n=1,2, \cdots ; \Phi_{-i}(x) \equiv 0 ; A_{i} \text { are constants }\right)
\end{aligned}
$$

with the following properties: (i) the zeros $c_{1, n}<c_{2, n}<\cdots<c_{n, n}$ of each $\omega_{n}$ are real and distinct; (ii) each $\omega_{n}$ generates an $F M Q$ formula (11). In discussing the convergence of (11), i.e., the relation $\lim _{n \rightarrow \infty} R_{n}(f)=0$, we consider two cases.

Case I. All abscissas belong to $[a, b]$. Here we have

TheOREM X. The FMQ formula (11), with all abscissas in $[a, b]$, converges for any bounded $f(x)$ for which $\int_{a}^{b} f(x) d \psi$ exists.

This is a direct extension of an identical theorem of Fejér stated for the special case of the ordinary Riemann integral $\int_{a}^{b} f(x) d x *^{*}$ The present case requires more care, due to possible discontinuities of $\psi(x)$. $\dagger$ Let $F$ denote the class of functions to which our $F M Q$ formula can be applied. Thus, the statement $f(x) \subset F$ implies that $\int_{a}^{b} f(x) d \psi$ exists and

* Fejér, loc. cit., pp. 303-307. A different proof for this special case was given earlier by Stekloff (Bulletin of the Russian Academy of Sciences, 1916). The same result was derived recently by R. Bailey, Convergence of sequences of positive linear functional operators, Duke Mathematical Journal, vol. 2 (1936), pp. 287-303.

$\dagger$ The following version of the proof is due to J. D. Tamarkin. It is identical in principle with, but is an improvement in form over, the original proof of the author. 


$$
Q_{n}(f) \rightarrow \int_{a}^{b} f(x) d \psi, \text { as } n \rightarrow \infty .
$$

We now have the following

LEMMA. If $\int_{a}^{b} f(x) d \psi$ exists and if, corresponding to any $\epsilon>0$, there exist two functions $f_{1, \epsilon}(x)$ and $f_{2, e}(x)$, both belonging to $F$ and such that

$$
f_{1, \epsilon}(x) \leqq f(x) \leqq f_{2, \epsilon}(x) \text { in }[a, b], 0 \leqq \int_{a}^{b}\left[f_{2, \epsilon}(x)-f_{1, \epsilon}(x)\right] d \psi<\epsilon,
$$

then also $f(x) \subset F$.

In fact, due to the positiveness of the coefficients $C_{i}$ in

$$
Q_{n}(f) \equiv \sum_{i=1}^{n} C_{i} f\left(c_{i}\right)
$$

we have for each $n$,

$$
Q_{n}\left(f_{1, \epsilon}\right) \leqq Q_{n}\left(f_{2, \epsilon}\right) .
$$

Letting here $n \rightarrow \infty$ and observing that, by hypothesis,

$$
Q_{n}\left(f_{j, \epsilon}\right) \rightarrow \int_{a}^{b} f_{j, \epsilon}(x) d \psi, \quad j=1,2,
$$

we conclude that both $\lim \sup _{n \rightarrow \infty} Q_{n}(f)$ and $\lim \inf _{n \rightarrow \infty} Q_{n}(f)$ lie between $\int_{a}^{b} f_{1, \epsilon}(x) d \psi$ and $\int_{a}^{b} f_{2, \epsilon}(x) d \psi$, each of which, tends to $\int_{a}^{b} f(x) d \psi$, as $\epsilon \rightarrow 0$. It follows that

$$
Q_{n}(f) \rightarrow \int_{a}^{b} f(x) d \psi, \text { as } n \rightarrow \infty,
$$

and this proves our Lemma.

In order to prove Theorem $\mathrm{X}$, we observe that all polynomials belong to $F$. Since any continuous function $f(x)$ can be approximated uniformly on $[a, b]$ by polynomials, the above lemma shows that all continuous functions also belong to $F$. Now let $f(x)$ be a step-function taking the constant value 1 in $(\alpha, \beta)(a<\alpha<\beta<b)$ and the value zero elsewhere in $[a, b]$, where $\alpha$ and $\beta$ are points of continuity of $\psi(x)$. Let $\delta<b-a$ be such that $a<\alpha-\delta, \beta+\delta<b$. Define two continuous functions $f_{1, \delta}(x), f_{2, \delta}(x)$ as follows:

$$
\begin{aligned}
& f_{1, \delta}(x)=\left\{\begin{array}{l}
1 \text { in }[\alpha, \beta], 0 \text { in }[a, \alpha-\delta],[\beta+\delta, b], \\
\text { linear in }(\alpha-\delta, \alpha),(\beta, \beta+\delta),
\end{array}\right. \\
& f_{2, \hat{o}}(x)=\left\{\begin{array}{l}
1 \text { in }[\alpha+\delta, \beta-\delta], 0 \text { in }[a, \alpha],[\beta, b], \\
\text { linear in }(\alpha, \alpha+\delta),(\beta-\delta, \beta) .
\end{array}\right.
\end{aligned}
$$


It is clear that

On the other hand,

$$
f_{1, \delta}(x) \leqq f(x) \leqq f_{2, \delta}(x) \text { in }[a, b] .
$$

$0 \leqq \int_{a}^{b}\left[f_{2, \delta}(x)-f_{1, \delta}(x)\right] d \psi \leqq \psi(\alpha+\delta)-\psi(\alpha-\delta)+\psi(\beta+\delta)-\psi(\beta-\delta)$.

Since $\alpha, \beta$ are points of continuity of $\psi(x)$, the right-hand member tends to 0 as $\delta \rightarrow 0$; hence, by the above lemma, $f(x) \subset F$. It follows that any linear combination of a finite number of functions of the type of the above $f(x)$ belongs to $F$, and so does, therefore, any step-function with a finite number of steps, whose points of discontinuity in $(a, b)$ are points of continuity of $\psi(x)$. (The values of such a function at its points of discontinuity may be chosen arbitrarily, since they do not affect the value of $\int_{a}^{b} f(x) d \psi$.) that

Assume now that $f(x)$ is any function for which $\int_{a}^{b} f(x) d \psi$ exists. This means

$$
\int_{a}^{b} f(x) d \psi=\lim \sum_{i=0}^{n-1} f\left(\xi_{i}\right)\left[\psi\left(x_{i}\right)-\psi\left(x_{i-1}\right)\right], \text { as } \max \left(x_{i}-x_{i-1}\right) \rightarrow 0 .
$$

Here $x_{0}=a, x_{n}=b, x_{i-1}<x_{i}$, and $\xi_{i}$ is taken arbitrarily in $\left[x_{i-1}, x_{i}\right]$. Since the set of points of continuity of $\psi(x)$ is dense in $[a, b]$, we may assume all interior points of subdivision $x_{1}, x_{2}, \cdots, x_{n-1}$ to be points of continuity of $\psi(x)$. Let $m_{i}, M_{i}$ denote respectively $\inf _{x} f(x), \sup _{x} f(x)$ for $x$ in $\left[x_{i-1}, x_{i}\right]$. Let $f_{1, n}(x), f_{2, n}(x)$ be two step-functions assuming constant values $m_{i}, M_{i}$ respectively in $\left[x_{i-1}, x_{i}\right]$, their values at $x=x_{i}$ being arbitrary, subject to the only condition

$$
f_{1, n}\left(x_{i}\right) \leqq f\left(x_{i}\right), \quad f_{2, n}\left(x_{i}\right) \geqq f\left(x_{i}\right), \quad i=1,2, \cdots, n-1 .
$$

Then it is clear that

$$
\begin{aligned}
& f_{1, n}(x) \leqq f(x) \leqq f_{2}(x), \quad a \leqq x \leqq b, \\
& \int_{a}^{b} f_{i, n}(x) d \psi \rightarrow \int_{a}^{b} f(x) d \psi, \text { as } n \rightarrow \infty, \quad j=1,2 .
\end{aligned}
$$

A direct application of the lemma shows that $f(x) \subset F$, and this completes the proof of our theorem.

Remark. For $A_{1}=A_{2}=\cdots=A_{k-1}=0$, the preceding proof shows the convergence of any $G M Q$ formula (in a finite interval), without imposing any restriction on $\psi(x)$.*

* Stieltjes in his classical paper, Quelques recherches sur les quadratures dites mécaniques, Oeuvres, vol. I, pp. 377-394, has proved the convergence of the GMQ formula for the case $d \psi(x)=p(x) d x$, $p(x) \geqq 0$, with the restriction $\int_{\alpha}^{\beta} p(x) d x>0, a \leqq \alpha<\beta \leqq b$. It was the desire to lift this restriction that prompted Stieltjes to introduce a new concept of integral which we now call the Stieltjes Integral. 
Case II. Some abscissas fall outside $[a, b]$. Without loss of generality, we may assume $a=-1, b=1$.

THEOREM XI. The convergence Theorem $\mathrm{X}$ for the $F M Q$ formula generated by the polynomial

$$
\omega_{n}(x)=\Phi_{n}(x)+A_{1} \Phi_{n-1}(x)+\cdots+A_{k-1} \Phi_{n-k+1}(x)
$$

holds, regardless of the location of the abscissas relative to $(-1,1)$, even if the coefficients $A_{1}, \cdots, A_{k-1}$ and the integer $k$ vary with $n$, provided: (i) $\psi(x)$ is continuous at $x= \pm 1$, and (ii) the number $l$ of abscissas $c_{i, n}$ such that $1 \leqq\left|c_{i, n}\right|<1$ $+h$, where $h>0$ is arbitrarily small but fixed, is o $\left[K_{\sigma}( \pm 1 ; d \psi)\right], \sigma=[(2 n-k) / 2]$. (The latter condition is obviously satisfied if $k$ is bounded, for $K_{\sigma}( \pm 1 ; d \psi)$ $\rightarrow \infty$, as $n \rightarrow \infty$; see Lemma I below.)

An analysis of the proof of Theorem $\mathrm{X}$, in connection with our agreement concerning $f\left(c_{i, n}\right)$ for $\left|c_{i, n}\right|>1$, shows at once that Theorem XI will be proved if we prove the following lemmas.

LEMMA I. Let $c$ denote an end-point of $[a, b]$, where $\psi(x)$ is continuous. Then

$$
\lim _{n \rightarrow \infty} K_{n}(c ; d \psi)=\infty .
$$

Reduce, without loss of generality, $[a, b]$ to $[-1,1]$. Take, to be definite, $c=1$. Then (M, p. 52)

$$
\begin{aligned}
\frac{1}{K_{n}(1 ; d \psi)} & \leqq \int_{0}^{1} x^{2 n} d \psi=\left(\int_{0}^{1-n-2 / 3}+\int_{1-n^{-2 / 3}}^{1}\right) x^{2 n} d \psi \\
& =o(1)+\int_{1-n^{-2 / 3}}^{1} d \psi(x) \\
& =o(1)+\psi(1)-\psi\left(1-n^{-2 / 3}\right) \rightarrow 0, \text { as } n \rightarrow \infty
\end{aligned}
$$

Combining the above lemma with Theorem IX, we derive

LEMMA II. If $c$, with the same significance and property as in Lemma I, is one of the abscissas, then the corresponding coefficient, say, $C_{i, n} \rightarrow 0$, as $n \rightarrow \infty$.

LEMMA III. Under the conditions of Theorem XI, the part of the summation $Q_{n}(f)$ which extends over abscissas not in $[-1,1]$ tends to 0 , as $n \rightarrow \infty$, if $f(x)$ is a polynomial of arbitrarily fixed degree r. Hence, making use of Lemma II,

$$
\sum_{\left|c_{i, n}\right|<1} C_{i, n} G_{r}\left(c_{i, n}\right) \rightarrow \int_{a}^{b} G_{r}(x) d \psi \text {, as } n \rightarrow \infty \quad(r \text { fixed }) .
$$

For brevity denote by $\xi$ any abscissa not in $[-1,1]$, with the correspond- 
ing coefficient $C_{\xi}$. We have, by Theorem IX,

$$
C_{\xi} \leqq \frac{1}{K_{\sigma}(\xi ; d \psi)}<\left\{\frac{2 \alpha_{0}^{1 / 2}}{\left[\xi+\left(\xi^{2}-1\right)^{1 / 2}\right]^{\sigma}+\left[\xi-\left(\xi^{2}-1\right)^{1 / 2}\right]^{\sigma}}\right\}^{2},|\xi|>1 .^{*}
$$

Moreover, according to Tchebyheff,

$$
\begin{aligned}
& \left|G_{r}(x)\right| \leqq g \text { on }[-1,1] \text { implies } \\
& \left|G_{r}(\xi)\right| \leqq \frac{g}{2}\left|\left[\xi+\left(\xi^{2}-1\right)^{1 / 2}\right]^{r}+\left[\xi-\left(\xi^{2}-1\right)^{1 / 2}\right]^{r}\right|, \quad|\xi|>1 .
\end{aligned}
$$

Hence,

$$
\begin{aligned}
\left|C_{\xi} G_{r}(\xi)\right| & \leqq \frac{4 g \alpha_{0}}{\left[\xi+\left(\xi^{2}-1\right)^{1 / 2}\right]^{2 \sigma-r}}, \quad\left|\xi+\left(\xi^{2}-1\right)^{1 / 2}\right|>1, \\
\left|\sum_{|\xi| \geqq 1+h} C_{\xi} G_{r}(\xi)\right| & \leqq 4 g k \alpha_{0}\left\{1+h+\left[(1+h)^{2}-1\right]^{1 / 2}\right\}^{r-2 \sigma} .
\end{aligned}
$$

Furthermore,

$$
C_{\xi} \leqq \frac{1}{K_{\sigma}(\xi ; d \psi)} \leqq \frac{1}{K_{\sigma}(d ; d \psi)}, \quad \text { if } \quad 1 \leqq|\xi|<1+h, \dagger
$$

where $d$ is that of the points \pm 1 which is nearest to $\xi$, and

$$
\begin{aligned}
\left|\sum_{1 \leqq|\xi|<1+h} C_{\xi} G_{r}(\xi)\right| & \leqq \frac{l \bar{g}}{K_{\sigma}(1 ; d \psi)}+\frac{l \bar{g}}{K_{\sigma}(-1 ; d \psi)}, \\
\bar{g} & =\max \left|G_{r}(x)\right| \text { in }[-1-h, 1+h] .
\end{aligned}
$$

Upon combining (39) and (41), we obtain a proof of our Lemma.

Remark. If we assume that the inequality $\left|c_{i, n}\right| \geqq 1+h$ holds for all abscissas outside $(-1,1)$ (i.e., the number $l$ in Theorem XI is zero), then the condition that $\psi(x)$ be continuous at $x= \pm 1$ is unnecessary, as seen from (39). It is interesting to note that the same reasoning which yields the proof of Theorem $\mathrm{X}$ proves also the following

THEOREM XII. Let $\left\{\psi_{n}(x)\right\}, n=1,2, \cdots$, be a sequence of functions monotonic and uniformly bounded in the finite interval $[a, b]$, such that, as $n \rightarrow \infty$,

* J. Shohat, On a general formula in the theory of Tchebycheff polynomials and its applications, these Transactions, vol. 29 (1927), pp. 569-583; p. 575. The considerations therein employed for $d \psi(x)=q(x) d x$, apply, without modification, to the general $d \psi(x)$.

$\dagger K_{n}^{\prime}(x)=2 \sum_{i=1}^{n} \phi_{i}(x) \phi_{i}^{\prime}(x)>0, x>x_{n, n}$, and $<0, x<x_{1, n}$, so that, as $x$ increases, $K_{n}(x)$ increases for $x>x_{n, n}$ and decreases for $x<x_{1, n}$. 
$\psi_{n}(x)$ converges to a monotonic function $\psi(x)$ over a set of points dense in $[a, b]$ and containing $a, b$. Then

$$
\lim _{n \rightarrow \infty} \int_{a}^{b} f(x) d \psi_{n}(x)=\int_{a}^{b} f(x) d \psi(x)
$$

for any $f(x)$ for which $\int_{a}^{b} f(x) d \psi_{n}(x), \int_{a}^{b} f(x) d \psi(x)$ exist.

This constitutes an extension of the Helly-Bray Theorem, ${ }^{*}$ where $f(x)$ is assumed to be continuous, but, to compensate, $\psi(x), \psi_{n}(x)$ are functions of bounded variation.

8. New representation of $C_{i}$ and $Q_{n}(f)$; relation to algebraic continued fractions. Given the $L M Q$ formula (11), introduce the polynomial of degree $n-1$,

$$
\sigma_{n}(x)=\int_{-1}^{1} \frac{\omega_{n}(x)-\omega_{n}(y)}{x-y} d \psi(y),
$$

and we get the following representation for $C_{i}, Q_{n}(f)$ :

$$
C_{i}=\frac{\sigma_{n}\left(c_{i}\right)}{\omega_{n}^{\prime}\left(c_{i}\right)}, \quad i=1,2, \cdots, n ; \quad Q_{n}(f)=\sum_{i=1}^{n} \frac{\sigma_{n}\left(c_{i}\right)}{\omega_{n}^{\prime}\left(c_{i}\right)} f\left(c_{i}\right) .
$$

In particular, for any fixed $z$, real or complex, but not on $[-1,1]$, we have, assuming that all $c_{i}$ are on $[-1,1]$ :

$$
Q_{n}\left(\frac{1}{z-x}\right)=\frac{\sigma_{n}(z)}{\omega_{n}(z)} \text {. }
$$

Returning to $F M Q$ formulae, we observe that our convergence theorem evidently holds for complex functions of the real variable $x$, hence, also for the function $1 /(z-x), z$ being fixed as in (44). Thus, for the $F M Q$ formula as described in Theorem $\mathrm{X}$, with all abscissas on $[-1,1]$,

$$
\lim _{n \rightarrow \infty} \frac{\sigma_{n}(z)}{\omega_{n}(z)}=\int_{-1}^{1} \frac{d \psi(x)}{z-x},
$$

$z$ given, real or complex, not on $[-1,1]$.

Now introduce a sequence of horizontal step-functions $\psi_{n}(x), n=1,2, \cdots$, as follows: $\psi_{n}(-1)=0, \psi_{n}(x)$ is constant in each interval $\left[-1, c_{1, n}\right)$, $\left[c_{1, n}, c_{2, n}\right), \cdots,\left[c_{n, n}, 1\right]$, and has a saltus at $x=c_{1, n}, c_{2, n}, \cdots, c_{n, n}$, the saltus at $x=c_{i, n}$ being $C_{i, n}, i=1,2, \cdots, n$. It follows that $\psi_{n}(x)$ is bounded and

* Theorem XII is known to hold for continuous $f(x)$ (cf. G. C. Evans, The Logarithmic Potential, American Mathematical Society Colloquium Publications, vol. 6, pp. 14-15), and the above reasoning is applicable, for $\int_{a}^{b} f(x) d \psi_{n}(x)$, which here replaces $Q_{n}(f)$, has the same positive linear character as the latter. 
non-decreasing in $[-1,1]$, with $\psi_{n}(1)=C_{1, n}+\cdots+C_{n, n}=\alpha_{0}$. With the aid of the sequence $\left\{\psi_{n}(x)\right\}$, we may rewrite our $F M Q$ formula (11) as

$$
\int_{-1}^{1} f(x) d \psi(x)=\int_{-1}^{1} f(x) d \psi_{n}(x)+R_{n}(f)
$$

and our convergence Theorem $\mathrm{X}$ states that

$$
\lim _{n \rightarrow \infty} \int_{-1}^{1} f(x) d \psi_{n}(x)=\int_{-1}^{1} f(x) d \psi(x) .
$$

It is interesting to note that (47) has been obtained without further investigating the nature of $\psi_{n}(x)$.

The application of the above considerations to $G M Q$ formulae is especially interesting. Here $\sigma_{n}(z) / \omega_{n}(z) \equiv \Omega_{n}(z) / \Phi_{n}(z)$ is the $(n+1)$ st convergent to the continued fraction

$$
\frac{\lambda_{1} \mid}{\mid z-c_{1}}-\frac{\lambda_{2} \mid}{\mid z-c_{2}}-\cdots-\frac{\lambda_{n} \mid}{\mid z-c_{n}}-\cdots
$$

"associated" with the integral $\int_{-1}^{1} d \psi(y) /(z-y)$, and thus (47) yields at once the convergence of the continued fraction (48) to the above integral, for any $z$ real or complex, not on $[-1,1]$. $^{*}$

9. The remainder. Write

$$
R_{n}(f)=\int_{-1}^{1}\left[f(x)-G_{q}(x)\right] d \psi-\sum_{i=1}^{n} C_{i, n}\left[f\left(c_{i, n}\right)-G_{q}\left(c_{i, n}\right)\right],
$$

and consider several cases.

(i) $f(x)$ is continuous in $[-1,1]$. (49) gives at once:

$$
\left|R_{n}(f)\right|<2 \alpha_{0} E_{q}(f)
$$

where $E_{q}(f)$ is the "best approximation" of $f(x)$ on $[-1,1]$ by polynomials of degree $\leqq q$, i.e.,

$$
E_{q}(f)=\min _{G_{q}} \max _{|x| \leqq 1}\left|f(x)-G_{q}(x)\right| .
$$

In particular, with $f(x)=1 /(z-x), z$ real and $|z|>1$, and all $c_{i, n}$ on $[-1,1]: \dagger$

* The same convergence theorem shows immediately that the "moment-problem"

$$
\int_{a}^{b} x^{n} d \psi(x)=\text { given } \alpha_{n}, n=0,1, \cdots,
$$

for the finite interval $(a, b)$ is "determined," i.e., has at most one solution, for any one solution gives rise to the same sequence of $O P$, hence, to the same $G M Q$ formula.

$\dagger$ The expression for $E_{n}(1 /(z-x))$ is due to S. Bernstein (Leçons sur les Propriétes Extrémales et la Meilleure Approximation des Fonctions Analytiques d'une Variable Réelle, Paris, 1926, p. 121). 


$$
\begin{aligned}
\left|R_{n}\left(\frac{1}{z-x}\right)\right| & \equiv\left|\int_{-1}^{1} \frac{d \psi(x)}{z-x}-\frac{\sigma_{n}(z)}{\omega_{n}(z)}\right| \\
& <2 \alpha_{0} /\left(z^{2}-1\right)\left[z+\left(z^{2}-1\right)^{1 / 2}\right]^{q}\left(\left|z+\left(z^{2}-1\right)^{1 / 2}\right|>1\right) .
\end{aligned}
$$

(More generally, (49) shows the convergence, for any continuous $f(x)$, of every $L M Q$ formula, for which the $\operatorname{sum} \sum_{i=1}^{n}\left|C_{i, n}\right|$ is bounded, as $n \rightarrow \infty$.) For $q=2 n-1,(51)$ gives the degree of convergence of the continued fraction (48) to $\int_{-1}^{1} d \psi(x) /(z-x)$ for real $z$, outside $[-1,1]$.

If, in addition, $f(x)$ has in $[-1,1]$ derivatives of various orders, we may take in (49) for $G_{q}(x)$ a properly chosen interpolation polynomial for $f(x)$. Thus, with $q=2 n-4, s_{1}=s_{2}=1, c_{1, n}=-1, c_{n, n}=1$, choose $G_{q}(x)$ so that

$G_{q}( \pm 1)=f( \pm 1), \quad G_{q}^{(\alpha)}\left(c_{i, n}\right)=f^{(\alpha)}\left(c_{i, n}\right), \quad \alpha=0,1 ; i=2,3, \cdots, n-1$.

Then, by virtue of (23),

$$
R_{n}(f)=-\frac{f^{(2 n-2)}(\xi)}{(2 n-2) !}\left\{\frac{1}{a_{n-1}^{2}\left(d \psi_{1}\right)}+\frac{B_{1}}{a_{n-3}^{2}\left(d \psi_{1}\right)}\right\},
$$

where $|\xi| \leqq 1$ and $d \psi_{1}=\left(1-x^{2}\right) d \psi$.

(ii) $f(x)$ is analytic. Let $f(t)(t=x+i y)$ be analytic in a certain region, bounded by a simple closed rectifiable curve $C$ which contains in its interior the line-segment $[-1,1]$. Then

$$
f(x)=\frac{1}{2 \pi i} \int_{c} \frac{f(t) d t}{t-x}
$$

and, by (44),

$$
\int_{-1}^{1} f(x) d \psi(x)=\frac{1}{2 \pi i} \int_{-1}^{1} d \psi(x) \int_{C} \frac{f(t) d t}{t-x}=\frac{1}{2 \pi i} \int_{C} \frac{f(t) \sigma_{n}(t)}{\omega_{n}(t)} d t+R_{n}(f) .
$$

It is readily proved that

$$
\int_{-1}^{1} d \psi(x)\left\{\int_{\alpha}^{\beta} F(x, y) d y\right\}=\int_{\alpha}^{\beta} d y\left\{\int_{-1}^{1} F(x, y) d \psi(x)\right\}
$$

if $F(x, y)$ is continuous in $x, y$ for $-1 \leqq x \leqq 1 ; \alpha \leqq y \leqq \beta ;(\alpha, \beta)$ finite. Hence,

$$
\begin{gathered}
\frac{1}{2 \pi i} \int_{-1}^{1} d \psi(x) \int_{C} \frac{f(t) d t}{t-x}=\frac{1}{2 \pi i} \int_{C} f(t) d t \int_{-1}^{1} \frac{d \psi(x)}{t-x} \\
R_{n}(f)=\frac{1}{2 \pi i} \int_{C} f(t)\left[\int_{-1}^{1} \frac{d \psi(x)}{t-x}-\frac{\sigma_{n}(t)}{\omega_{n}(t)}\right] d t .
\end{gathered}
$$


We proceed to transform the expression (56). Denoting generally

$$
\frac{\alpha^{\prime}}{x^{s}}+\frac{\beta^{\prime}}{x^{s+1}}+\cdots=\left(\frac{1}{x^{s}}\right), \quad s>0, \quad \alpha^{\prime} \neq 0,
$$

we have, by the definition of degree of precision,

$$
\begin{aligned}
\omega_{n}(t) F(t)-\sigma_{n}(t) & =\left(\frac{1}{x^{q-n+2}}\right), \quad F(t)=\int_{-1}^{1} \frac{d \psi(x)}{t-x}, \\
F(x)-\frac{\sigma_{n}(t)}{\omega_{n}(t)} & =\left(\frac{1}{x^{q+2}}\right) .
\end{aligned}
$$

Upon writing

$$
\frac{\sigma_{n}(t)}{\omega_{n}(t)}=\frac{1}{\omega_{n}(t)} \int_{-1}^{1} \frac{\omega_{n}(t)-\omega_{n}(x)}{t-x} d \psi(x)=F(t)-\frac{1}{\omega_{n}(t)} \int_{-1}^{1} \frac{\omega_{n}(x) d \psi(x)}{t-x}
$$

and comparing with (57), we conclude that

$$
\begin{aligned}
F(t)-\frac{\sigma_{n}(t)}{\omega_{n}(t)} & =\frac{1}{\omega_{n}(t)} \int_{-1}^{1} \frac{\omega_{n}(x) d \psi(x)}{t-x} \equiv \frac{S_{n}(t)}{\omega_{n}(t)} \\
S_{n}(t) & =\int_{-1}^{1} \frac{\omega_{n}(x) d \psi(x)}{t-x}
\end{aligned}
$$

and (56) gives

$$
R_{n}(f)=\frac{1}{2 \pi i} \int_{C} \frac{f(t) S_{n}(t) d t}{\omega_{n}(t)}=\frac{1}{2 \pi i} \int_{C} f(t)\left[F(t)-\frac{\sigma_{n}(t)}{\omega_{n}(t)}\right] d t .
$$

In the special case of the GMQ formula, $S_{n}(t)$ is the so-called Tchebycheff function of second kind,

$$
S_{n}(t)=\int_{-1}^{1} \frac{\Phi_{n}(x) d \psi(x)}{t-x}
$$

and (59) gives an expression for the remainder of the GMQ formula in terms of the remainder of the associated continued fraction (48). Namely,

$$
R_{n}(f)=\frac{1}{2 \pi i} \int_{C} \frac{f(t) S_{n}(t) d t}{\Phi_{n}(t)}=\frac{1}{2 \pi i} \int_{C} f(t)\left[F(t)-\frac{\Omega_{n}(t)}{\Phi_{n}(t)}\right] d t
$$

where, we recall, $\Omega_{n}(z) / \Phi_{n}(z)$ is the $(n+1)$ st convergent to (48). In the general case of an $F M Q$ formula with degree of precision $q=2 n-k$, we may substitute into (42) the expression (14) for $\omega_{n}(x)$, which gives, by (60), 


$$
\begin{aligned}
R_{n}(f) & =\frac{1}{2 \pi i} \int_{C} f(t) \frac{S_{n}(t)+A_{1} S_{n-1}(t)+\cdots+A_{k-1} S_{n-k+1}(t)}{\Phi_{n}(t)+A_{1} \Phi_{n-1}(t)+\cdots+A_{k-1} \Phi_{n-k+1}(t)} d t \\
& =\frac{1}{2 \pi i} \int_{C} f(t)\left\{F(t)-\frac{\Omega_{n}(t)+A_{1} \Omega_{n-1}(t)+\cdots+A_{k-1} \Omega_{n-k+1}(t)}{\Phi_{n}(t)+A_{1} \Phi_{n-1}(t)+\cdots+A_{k-1} \Phi_{n-k+1}(t)}\right\} d t .
\end{aligned}
$$

Formulae (56), (61) hold for any $L M Q$ formula with all abscissas on [-1,1], $f(t)$ being analytic inside and on $C$.

The above formulae show once more the close connection between the theory of mechanical quadratures and that of algebraic continued fractions. The latter enables us to estimate $R_{n}(f)$ if an estimate is known for $S_{n}(t)$.

For real $t$ we may proceed as follows. Formula (60) shows that the $\left\{S_{n}(t)\right\}$ are the coefficients in the expansion

$$
\frac{1}{t-x} \sim \sum_{n=0}^{\infty} a_{n} S_{n}(t) \phi_{n}(x)
$$

whence, from known results,

$$
\left|S_{n}(t)\right| \leqq \alpha_{0}{ }^{1 / 2} / a_{n} E_{n}\left(\frac{1}{t-x}\right) \leqq \alpha_{0} /\left\{2^{n-1}\left(t^{2}-1\right)\left|t \pm\left(t^{2}-1\right)^{1 / 2}\right|^{n-1}\right\} .
$$

Here we assume that $t$ is real, $|t|>1$, and \pm is so chosen that $\left|t \pm\left(t^{2}-1\right)^{1 / 2}\right|$ $>1$. Furthermore, if $\delta$ denotes the minimum distance from $t$ to the line-segment $[-1,1]$, then

$$
\left|\Phi_{n}(t)\right| \equiv\left|\left(t-x_{1, n}\right) \cdots\left(t-x_{n, n}\right)\right|>\delta^{n}
$$

whence,

$$
\left|F(t)-\frac{\Omega_{n}(t)}{\Phi_{n}(t)}\right| \equiv\left|\frac{S_{n}(t)}{\Phi_{n}(t)}\right| \leqq \alpha_{0} / 2^{n-1}\left(t^{2}-1\right) \delta^{n}\left|t \pm\left(t^{2}-1\right)^{1 / 2}\right|^{n-1} .
$$

10. The case of $d \psi(x)=p(x) d x . p(x)$ an $S$-function. The Lebesgue integral

$$
\int_{-1}^{1} \frac{p(x) d x}{\left(1-x^{2}\right)^{1 / 2}}
$$

then exists. In this important special case we can go much further in the discussion of the abscissas and the remainder in our $F M Q$ formula (11), for here $\Phi_{n}(x)$ possesses many asymptotic properties. Thus, as $n \rightarrow \infty$,

$$
a_{n}=2^{n} A[1+o(1)], \lambda_{n} \rightarrow \frac{1}{4}, c_{n} \rightarrow 0 ; A>0, \text { independent of } n .
$$

$$
\Phi_{n}( \pm 1)=o\left[\left(\frac{1}{2}+\epsilon\right)^{n}\right], \epsilon>0 \text {, arbitrarily small; }
$$

$$
\Phi_{n}( \pm 1) / \Phi_{n-1}( \pm 1) \rightarrow \pm \frac{1}{2} .
$$




$$
\begin{array}{r}
\Phi_{n}(x) \sim w^{n} \Phi(w), K_{n}(x) \sim(2 w)^{2 n} K(w) ; \Phi(w), K(w) \text { independent of } n, \\
w=\left\{x+\left(x^{2}-1\right)^{1 / 2}\right\} / 2,
\end{array}
$$

$x$ is not on $[-1,1](\mathrm{M}$, pp. 50, 52, 54). The determination of the radical $\left(x^{2}-1\right)^{1 / 2}$ here and hereafter is so chosen that $|w| \rightarrow \infty$, as $|x| \rightarrow \infty$. These asymptotic relations for $\Phi_{n}(x)$ hold uniformly in any finite closed region in the $x$-plane whose minimum distance from the line-segment $[-1,1]$ is positive. We denote such a region by $D$.

Now, the relations

$$
\frac{\Omega_{n}(x)}{\Phi_{n}(x)} \rightarrow F(x), \quad \frac{S_{n}(x)}{\Phi_{n}(x)} \rightarrow 0, \quad \text { as } \quad n \rightarrow \infty \quad\left(F(x)=\int_{-1}^{1} \frac{d \psi(y)}{x-y}\right)
$$

imply

$$
\frac{\Omega_{n+1}(x)}{\Omega_{n}(x)} \rightarrow w, \quad \text { as } n \rightarrow \infty,
$$

uniformly in $D$. Note that the transformation

$$
w=\left\{x+\left(x^{2}-1\right)^{1 / 2}\right\} / 2, \quad x=\frac{1}{2}\{2 w+1 /(2 w)\}
$$

maps conformally the complex $x$-plane onto the $w$-plane outside the circle $|w| \leqq \frac{1}{2}$, so that the line-segment $[-1,1]$ corresponds to the circumference $|w|=\frac{1}{2}$, and the line-segments $(-\infty,-1]$ and $[1, \infty)$ correspond to the linesegments $\left(-\infty,-\frac{1}{2}\right]$ and $\left[\frac{1}{2}, \infty\right)$ respectively. We now proceed to investigate the zeros $c_{i, n}$ of the polynomial

$$
\omega_{n}(x)=\Phi_{n}(x)+A_{1} \Phi_{n-1}(x)+\cdots+A_{k-1} \Phi_{n-k+1}(x),
$$

under the following assumptions, which we call "assumptions $P$ ":

( $\alpha$ ) $k$ is fixed, independent of $n$;

( $\beta$ ) $A_{1}, A_{2}, \cdots, A_{k-1}$, if dependent on $n$, have finite limits $h_{1}, h_{2}, \cdots, h_{k-1}$, as $n \rightarrow \infty$.

Note in passing that, by virtue of $(\alpha),(\beta)$, we have here

$$
\lim _{n \rightarrow \infty} \frac{\sigma_{n}(x)}{\omega_{n}(x)}=\lim _{n \rightarrow \infty} \frac{\Omega_{n}(x)+A_{1} \Omega_{n-1}(x)+\cdots+A_{k-1} \Omega_{n-k+1}(x)}{\Phi_{n}(x)+A_{1} \Phi_{n-1}(x)+\cdots+A_{k-1} \Phi_{n-k+1}(x)}=F(x)
$$

at any point $x$ not on $[-1,1]$, which does not coincide with one of the zeros of the polynomial

$$
H(w) \equiv w^{k-1}+h_{1} w^{k-2}+\cdots+h_{k-1} \quad\left(w=\left\{x+\left(x^{2}-1\right)^{1 / 2}\right\} / 2\right) .
$$

We have

(69) $\omega_{n}(x)=\Phi_{n-k+1} F_{n}(x), F_{n}(x) \equiv \frac{\Phi_{n}(x)}{\Phi_{n-k+1}(x)}+A_{1} \frac{\Phi_{n-1}(x)}{\Phi_{n-k+1}(x)}+\cdots+A_{k-1}$, 
and the rational function $F_{n}(x)$ is analytic outside $(-1,1)$; its zeros outside $[-1,1]$ are zeros for $\omega_{n}(x)$, and vice versa, and its only singularities are poles in $(-1,1)$. The following asymptotic relations form the basis of the subsequent discussion.

As $n \rightarrow \infty$, we have uniformly in $D$, by virtue of (63), (64), (65),

$$
\begin{aligned}
& \frac{\omega_{n}(x)}{\Phi_{n-k+1}(x)} \equiv F_{n}(x) \rightarrow H(w), \quad \omega_{n}(x) \sim \Phi_{n-k+1}(x) H(w), \\
& \frac{\omega_{n}( \pm 1)}{\Phi_{n-k+1}( \pm 1)}=F_{n}( \pm 1) \rightarrow H\left( \pm \frac{1}{2}\right) .
\end{aligned}
$$

It follows that in all cases

$$
\omega_{n}( \pm 1) \rightarrow 0, \quad \text { as } \quad n \rightarrow \infty
$$

We set

$$
w_{i, n}=\left\{c_{i, n}+\left(c_{i, n}^{2}-1\right)^{1 / 2}\right\} / 2, \quad(i=1,2, \cdots, n ; n=1,2, \cdots)
$$

corresponding to the zeros of $\omega_{n}(x)$ (at least $n-k+1$ of which lie in $(-1,1)$ ). We denote by $w_{j}^{\prime}, j=1,2, \cdots, k-1$, the zeros of $H(w)$, and set

$$
x_{j}^{\prime}=\frac{1}{2}\left(2 w_{j}^{\prime}+\frac{1}{2 w_{j}^{\prime}}\right), \quad j=1,2, \cdots, k-1 .
$$

The relations (69), (70) lead to

Theorem XIII. Let $D$ be a finite closed region in the $x$-plane at a minimum positive distance from the line-segment $[-1,1]$, and let $D^{\prime}$ be the corresponding region in the w-plane. If $D^{\prime}$ contains in its interior zeros $w_{j_{1}}^{\prime}, w_{j_{2}}{ }^{\prime}, \cdots, w_{j_{\mu}}{ }^{\prime}$ of $H(w)$, but no zeros $w_{j}^{\prime}$ on the boundary $(k-1 \geqq \mu \geqq 0)$, then for $n \geqq N=N\left(D^{\prime}\right)$ sufficiently large, $\omega_{n}(x)$ has in $D^{\prime}$ precisely $\mu$ zeros $c_{j_{1}, n}, \cdots, c_{j_{\mu}, n}{ }^{\prime}$ (a zero of multiplicity $\rho$ being counted $\rho$ times). Moreover,

$$
\lim _{n \rightarrow \infty} c_{j_{s}, n}^{\prime}=x_{j_{s}}^{\prime}=\frac{1}{2}\left\{2 w_{j_{\iota}}^{\prime}+\frac{1}{2 w_{j_{\iota}}^{\prime}}\right\}, \quad s=1,2, \cdots, \mu .
$$

We readily draw the following conclusions:

(i) The zeros of $\omega_{n}(x), n=1,2, \cdots$, are all bounded.

(ii) Denote by $\nu$ the number of zeros $w_{j}^{\prime \prime}$ outside the circle $|w| \leqq 1 / 2$. For $n$ sufficiently large, $\omega_{n}(x)$ has precisely $\nu$ zeros $c_{i, n}^{\prime \prime}$ outside the line-segment $[-1,1]$ converging to $\frac{1}{2}\left\{2 w_{i}^{\prime \prime}+1 / 2 w_{i}^{\prime \prime}\right\}$, as $n \rightarrow \infty$. If $\nu=k-1$, these $c_{i, n}^{\prime \prime}$ account for all zeros of $\omega_{n}(x)$ outside $[-1,1]$. If $\nu<k-1$, the remaining zeros of $\omega_{n}(x)$, if they do not actually belong to $[-1,1]$, belong to it asymptotically, i.e., all their limit-points lie on $[-1,1]$. 
(iii) A necessary condition for the reality of all zeros of $\omega_{n}(x)$, for $n$ sufficiently large (hereafter expressed by $n \geqq N, N$ being properly chosen in the case under discussion) is that $H(w)$ shall not have imaginary zeros $w_{j}^{\prime}$, with $\left|w_{j}^{\prime}\right|>\frac{1}{2}$. In other words, if $\omega_{n}(x)$ generates an LMQ formula, then the zeros $w_{j}^{\prime}$ of $H(w)$ outside the circle $|w| \leqq \frac{1}{2}$ are necessarily all real. The corresponding points $x_{j}^{\prime}=\frac{1}{2}\left\{2 w_{j}^{\prime}+1 /\left(2 w_{j}^{\prime}\right)\right\}$ are the limiting positions, as $n \rightarrow \infty$, for the corresponding abscissas of our $L M Q$ formula. The most interesting case is that of $\omega_{n}(x)$ generating an $F M Q$ formula.

Theorem XIV. A necessary condition that

$$
\omega_{n}(x)=\Phi_{n}(x)+A_{1} \Phi_{n-1}(x)+\cdots+A_{k-1} \Phi_{n-k+1}(x)
$$

satisfying the "assumptions $P$ " generates an $F M Q$ formula for $n \geqq N$ is that all zeros of $H(w)$ lying outside the circle $|w| \leqq 1 / 2$ shall be real and that either interval $\left(-\infty,-\frac{1}{2}\right],\left[\frac{1}{2}, \infty\right)$ shall contain no more than $[k / 2]$ of these zeros.

As an illustration, we take up once more the special cases $k=2,3, A_{i}$ independent of $n$.

(i) $k=2$, i.e.,

$$
\omega_{n}(x)=\Phi_{n}(x)+A_{1} \Phi_{n-1}(x), \quad A_{1} \neq 0 .
$$

The discussion centers around $c_{1, n}, c_{n, n}$. Here

$$
H(w) \equiv w+A_{1}, \quad w_{1}^{\prime}=-A_{1} .
$$

$A_{1}>\frac{1}{2}$ implies $c_{1, n}<-1(n \geqq N), c_{1, n} \rightarrow \frac{1}{2}\left(2 A_{1}+1 /\left(2 A_{1}\right)\right)$, as $n \rightarrow \infty$.

$A_{1}<-\frac{1}{2}$ implies $c_{n, n}>1(n \geqq N), c_{n, n} \rightarrow \frac{1}{2}\left(2 A_{1}+1 /\left(2 A_{1}\right)\right)$, as $n \rightarrow \infty$.

$\left|A_{1}\right| \leqq \frac{1}{2}$ implies $-1<c_{i, n}<1, i=1,2, \cdots, n$.

$$
\lim _{n \rightarrow \infty} \omega_{n}( \pm 1) / \Phi_{n}( \pm 1)=A_{1} \pm \frac{1}{2}
$$

in all cases. ${ }^{*}$ We may add that since $x_{1, n} \rightarrow-1, x_{n, n} \rightarrow+1$, as $n \rightarrow \infty$, we get from Theorem VIII

$$
A_{1}>\frac{1}{2} \text { implies } c_{n, n} \rightarrow 1 ; A_{1}<-\frac{1}{2} \text { implies } c_{1, n} \rightarrow-1 ;
$$

$$
\left|A_{1}\right| \leqq \frac{1}{2} \text { implies } c_{1, n} \rightarrow-1, c_{n, n} \rightarrow 1 \text {. }
$$

(ii) $k=3$, i.e.,

$$
\omega_{n}(x)=\Phi_{n}(x)+A_{1} \Phi_{n-1}(x)+A_{2} \Phi_{n-2}(x), \quad A_{2} \neq 0 .
$$

* Write

$$
\omega_{n}(x)=\Phi_{n-1}(x)\left[\frac{\Phi_{n}(x)}{\Phi_{n-1}(x)}+A_{1}\right],
$$

and note that $\Phi_{n}(x) / \Phi_{n-1}(x)$ always increases with $x$ and $\rightarrow \pm \frac{1}{2}$ at $x= \pm 1$, as $n \rightarrow \infty$. 
The discussion centers around two of the zeros of $\omega_{n}(x)$. Here

$$
H(w) \equiv w^{2}+A_{1} w+A_{2} .
$$

We discuss the following possibilities.

$$
A_{1}^{2}-4 A_{2}<0, \quad\left|w_{j}^{\prime}\right|>\frac{1}{2}, \quad j=1,2 .
$$

$\omega_{n}(x)$ has two imaginary zeros, which converge to $\frac{1}{2}\left\{2 w_{j}^{\prime}+1 /\left(2 w_{j}^{\prime}\right)\right\}, j=1,2$.

$$
A_{1}{ }^{2}-4 A_{2}>0, \quad\left|w_{j}^{\prime}\right|>\frac{1}{2}, \quad j=1,2 .
$$

The zeros of $\omega_{n}(x)$ are all real, two lying outside $(-1,1)$ and converging to $\frac{1}{2}\left\{2 w_{j}^{\prime}+1 /\left(2 w_{j}^{\prime}\right)\right\}, j=1,2$. If $A_{2}>0$, then $s_{1}=2, s_{2}=0$, or $s_{1}=0, s_{2}=2$. If $A_{2}<0$, then $s_{1}=s_{2}=1$. Thus, under the assumption (78.2), $\omega_{n}(x)$ generates an $L M Q$ formula which becomes an $F M Q$ formula, if and only if $A_{2}<0$ (see case $(\beta), \S 5)$.

$$
A_{1}^{2}-4 A_{2}>0, \quad\left|w_{1}^{\prime}\right| \leqq \frac{1}{2}, \quad\left|w_{2}^{\prime}\right|>\frac{1}{2} .
$$

Here again all zeros of $\omega_{n}(x)$ are real, one necessarily outside $(-1,1)$ and converging to $\frac{1}{2}\left\{2 w_{2}^{\prime}+1 /\left(2 w_{2}^{\prime}\right)\right\}$.

$$
A_{1}^{2}-4 A_{2}>0, \quad\left|w_{j}^{\prime}\right| \leqq \frac{1}{2},
$$$$
j=1,2 .
$$

The zeros of $\omega_{n}(x)$, for $n \geqq N$, either all belong to $[-1,1]$, or else, the limitpoints of the sequence of the two exterior zeros are on $[-1,1]$.

$$
A_{1}^{2}-4 A_{2}=0, \quad\left|A_{1}\right|>1 \text {. }
$$

Here again all zeros of $\omega_{n}(x)$ are real, with two outside [-1, 1] and both converging to $-\left(A_{1}^{2}+1\right) /\left(2 A_{1}\right)$. There may be a double root, or we may have $s_{1}=2, s_{2}=0$, or $s_{1}=0, s_{2}=2 ; \omega_{n}(x)$ cannot generate an $F M Q$ formula.

In a similar manner we may discuss the case $k=4$.

In the above discussion we have encountered cases where $\omega_{n}(x)$ generates an $L M Q$ formula which cannot have all coefficients positive. However, we are dealing here with polynomials $\Phi_{n}(x)$ which possess special properties, and the question arises: do these special properties compensate for the presence of negative coefficients in our $M Q$ formula? The answer is in the affirmative, as is shown in

THEOREM XV. Consider the polynomial (14) $\omega_{n}(x)$, where, in addition to the "assumptions $P$," it is assumed: (i) the zeros $w_{j}^{\prime}$ of the polynomial (68) $H(w)$ are all real and distinct, $\left|w_{j}^{\prime}\right|>1 / 2$. If $\omega_{n}(x)$ generates an LMQ formula, the latter converges, as $n \rightarrow \infty$, for any $f(x)$ for which $\int_{-1}^{1} f(x) d \psi$ exists.

The proof follows the same lines as that of Theorems X, XI, making use of the following properties of the $M Q$ formula under discussion: 
(i) the number of negative coefficients is bounded;

(ii) $\omega_{n}(x) \sim w^{n-k+1} \omega(w), x$ real or complex, not on $[-1,1], \omega(w)$ independent of $n$;

(iii) $A, B$ denoting certain fixed finite quantities independent of $n$, we have:

$$
\begin{aligned}
\left|a^{(i)}-a^{(j)}\right| \geqq h_{1}>0, \quad\left|b^{(l)}-b^{(m)}\right| \geqq h_{1}>0,-1-h_{2} \geqq a^{(i)} \geqq A, & \\
1+h_{2} \leqq b^{i} \leqq & B \quad\left(n \geqq N ; h_{1}, h_{2} \text { independent of } n ;\right. \\
& \left.i, j=1,2, \cdots, s_{1} ; l, m=1,2, \cdots, s_{2} ; i \neq j ; l \neq m\right) .
\end{aligned}
$$

(i) is known; (ii) follows from (71), (72), (73); (iii) follows from the fact that all $a^{(i)}, b^{(l)}$ converge, as $n \rightarrow \infty$, to certain fixed points $\frac{1}{2}\left\{2 w_{j}^{\prime}+1 /\left(2 w_{j}^{\prime}\right)\right\}$.

(iv) $s=k-1$, so that $q=2 n-s-1$, hence (by Theorem VI), all interior coefficients are positive.

We have thus obtained a wide class of convergent $L M Q$ formulae, the existence of which is assured by the considerations developed above.

Remark. We may liberalize considerably the conditions imposed upon $H(w)$, without impairing the validity of Theorem XV. Thus, under (62), $\psi(x)$ is continuous at $x= \pm 1$, so that $K_{n}( \pm 1 ; d \psi) \rightarrow \infty$, as $n \rightarrow \infty$, and we could modify accordingly the condition requiring that all $\left|w_{j}^{\prime}\right|>\frac{1}{2}$, permitting a certain number (necessarily finite) of $a^{(i)}, b^{(j)}$ to converge to $\mp 1$ respectively (see (40), (41)). We shall not dwell here upon this and other possible modifcations, except the following one.

THEOREM XV (bis). Given any $d \psi(x)$ and an LMQ formula employing $s=s_{1}+s_{2}\left(s_{1}, s_{2}\right.$ fixed) arbitrarily preassigned abscissas, with degree of precision $q=2 n-s-1$. The formula converges for any $f(x)$ for which $\int_{-1}^{1} f(x) d \psi$ exists; $\psi(x)$ is supposed to be continuous at $x= \pm 1$, if these points (one or both) are among the preassigned abscissas.

In fact, referring to $\$ 4$, we see that the conditions (i), (ii), (iii), (iv), given above and the expressions (30) for the exterior coefficients hold for the $L M Q$ formula under discussion.

11. Tchebycheff inequalities for the coefficients of some classes of $F M Q$ formulae. These important inequalities, given without proof by Tchebycheff in 1874 for GMQ formulae, were proved in 1884 by Stieltjes and Markoff independently.* Markoff went somewhat further than Stieltjes, extending Tchebycheff inequalities to certain classes of $F M Q$ formulae. The following

* Stieltjes, loc. cit., pp. 384-392; A. Markoff, On Certain Applications of Algebraic Continued Fractions (in Russian), Thesis, St. Petersburg, 1884. It is curious to note that both use the same proof, namely, applying properly constructed $M Q$ formulae to suitably chosen polynomials. In what follows the proof is omitted. 
is taken, with slight modifications and extensions (in order to cover the cases $q=2 n-3,2 n-4)$ from Markoff's Thesis.

THEOREM XVI. The coefficients $C_{i}$ in an FMQ formula, with degree of precision $q=2 n-1,2 n-2$, or $q=2 n-3$ with $s_{1}+s_{2}=1,2$, or $q=2 n-3,2 n-4$, $s_{1}=s_{2}=1$, satisfy the following Tchebycheff inequalities:

(1) $\int_{a}^{c_{i}} d \psi(x)>C_{1}+C_{2}+\cdots+C_{i-1}, \quad i=2,3, \cdots, n$,

(2) $\int_{a}^{c_{i}} d \psi(x)<C_{1}+C_{2}+\cdots+C_{i}, \quad i=1,2, \cdots, n$,

(3) $\int_{c_{i}}^{b} d \psi(x)>C_{i+1}+\cdots+C_{n}, \quad i=2,3, \cdots, n$,

(4) $\int_{c_{i}}^{b} d \psi(x)<C_{i}+\cdots+C_{n}, \quad i=1,2, \cdots, n$,

(5) $\int_{c_{i}}^{c_{k}} d \psi(x)>C_{i+1}+\cdots+C_{k-1}, \quad i=1,2, \cdots, n-1 ; k=2,3, \cdots, n$,

(6) $\int_{c_{i}}^{c_{k}} d \psi(x)<C_{i}+\cdots+C_{k}, \quad i, k=1,2, \cdots, n$.

In particular,

(7) $\int_{a}^{c_{1}} d \psi(x)<C_{1}, \quad \int_{c_{n}}^{b} d \psi(x)<C_{n}$,

(8) $\int_{c_{i}}^{c_{i+1}} d \psi(x)>0, \quad i=1,2, \cdots, n-1$,

(9) $\int_{c_{i}}^{c_{i+2}} d \psi(x)>C_{i+1}, \quad i=1,2, \cdots, n-2$.

Remarks. (i) These inequalities hold for any $(a, b)$, finite or infinite.

(ii) If $c_{i} \neq a, b$, we may assign in the above inequalities to $\psi(x)$ at $x=c_{i}$ any value in $\left[\psi\left(c_{i}-0\right), \psi\left(c_{i}+0\right)\right]$. Thus, (8) may be rewritten as

(8.1) $\int_{c_{i}+0}^{c_{i+1}-0} d \psi(x)>0$,

$$
i=1,2, \cdots, n-1
$$

which shows that a subinterval of constancy of $\psi(x)$ cannot contain more than one abscissa - a property proved above in a different manner.

(iii) We agree to let $\psi(x)=\psi(a)$ or $\psi(x)=\psi(b)$ for $x \leqq a$ or $x \geqq b$ respectively. Then it is not necessary to take (with Markoff) $c_{1} \geqq a, c_{n} \leqq b$. 
(iv) The above inequalities hold for an $L M Q$ formula, with $q=2 n-3$ and $s_{1}=2$ or $s_{2}=2$ (then necessarily $s_{2}=0$ or $s_{1}=0$ respectively), with the following exceptions: $c_{1}<c_{2} \leqq a$ invalidates the inequalities

$$
\int_{a}^{c_{1}} d \psi(x)<C_{1}, \quad \int_{c_{1}}^{c_{2}} d \psi(x)>0
$$

$c_{n}>c_{n-1} \geqq b$ invalidates the inequalities

$$
\int_{c_{n}}^{b} d \psi(x)<C_{n}, \quad \int_{c_{n-1}}^{c_{n}} d \psi(x)>0 .
$$

In fact, by Theorem VI, $C_{1}<0$ or $C_{n}<0$ respectively, while the integrals on the left vanish, according to our agreement.

(v) For an $L M Q$ formula, with $q=2 n-4, s_{1}=2, s_{2}=0$, or $s_{1}=0, s_{2}=2$, the above inequalities hold, with the same possible exceptions as stated for $q=2 n-3$. By virtue of (32), $c_{1}<c_{2} \leqq a$ actually invalidates (79.1) if $A_{3}<0$ in (14), and $c_{n}>c_{n-1} \geqq b$ actually invalidates inequalities (79.2) if $A_{3}>0$.

(vi) Assuming all $C_{i}>0$ and all $c_{i}$ in $(a, b)$, choose

$$
a<\xi_{1}<\xi_{2}<\cdots<\xi_{n} \leqq b,
$$

so that

$$
C_{1}+C_{2}+\cdots+C_{i}=\int_{a}^{\xi_{i}} d \psi(x), \quad i=1,2, \cdots, n .
$$

It follows from the inequalities $(1,2)$ that

$$
a<c_{1}<\xi_{1}<c_{2}<\xi_{2}<\cdots<c_{n}<\xi_{n}=b .
$$

12. Discussion of the abscissas and the coefficients based on Theorem XI.* First, assume $q \leqq 2 n-4$. We make use of the inequality (8), from which follows an important property of the abscissas which we call " $C$-property," namely: a sub-interval $(c, d)$ of constancy of $\psi(x)$, contains, including its endpoints, at most one abscissa.

We now turn to Theorem XI. Suppose that for a certain fixed $i$ and with certain $l, m$, we have, as $n \rightarrow \infty$ :

(80.1) $c_{i, n}, c_{i+1, n}, \cdots, c_{i+l, n} \rightarrow \xi^{\prime}$,

$$
\begin{aligned}
& \text { (80.2) } c_{i-1, n} c_{i-2, n}, \cdots, c_{i-m, n} \rightarrow \xi^{\prime \prime}, \quad a \leqq \xi^{\prime \prime}<\xi^{\prime} \leqq b, \\
& \text { (80.3) }\left|c_{i+l+1, n}-\xi^{\prime}\right|, \quad\left|c_{i-m-1, n}-\xi^{\prime \prime}\right|>h>0,
\end{aligned}
$$

\footnotetext{
* The reasoning of the second part of this section generally follows that of Fejer, but the results are considerably modified in many respects, due to our dealing with $\psi(x)$ not necessarily continuous, and, a fortiori, not necessarily absolutely continuous.
} 


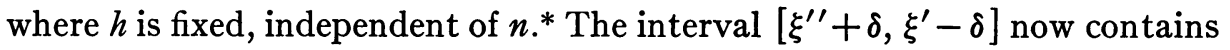
no zeros of $\omega_{n}(x)$, for $n \geqq N$. If $\xi^{\prime}, \xi^{\prime \prime}$ do not coincide with $b$ or $a$ respectively, choose $\delta$ so that $\xi^{\prime} \pm \delta, \xi^{\prime \prime} \pm \delta$ are points of continuity of $\psi(x)$. Apply Theorem $\mathrm{XI}$ to the following functions:

$$
\begin{aligned}
& f_{1}(x)=1 \text { in }\left[\xi^{\prime \prime}+\delta, \xi^{\prime}-\delta\right], f_{2}(x)=1 \text { in }\left[\xi^{\prime}-\delta, \xi^{\prime}+\delta\right], \\
& f_{3}(x)=1 \text { in }\left[\xi^{\prime \prime}-\delta, \xi^{\prime \prime}+\delta\right] ; f_{j}(x)=0 \text { elsewhere, } j=1,2,3 .
\end{aligned}
$$

We get:

$$
\begin{aligned}
& \lim _{n \rightarrow \infty} Q_{n}\left(f_{1}\right)=\int_{\xi^{\prime \prime}+\delta}^{\xi^{\prime}-\delta} d \psi(x)=\psi\left(\xi^{\prime}-\delta\right)-\psi\left(\xi^{\prime \prime}+\delta\right)=0, \\
& \lim _{n \rightarrow \infty} Q_{n}\left(f_{2}\right)=\int_{\xi^{\prime}-\delta}^{\xi^{\prime}+\delta} d \psi=\lim _{n \rightarrow \infty}\left(C_{i, n}+C_{i+1, n}+\cdots+C_{i+l, n}\right), \\
& \lim _{n \rightarrow \infty} Q_{n}\left(f_{3}\right)=\int_{\xi^{\prime \prime}-\delta}^{\xi^{\prime \prime}+\delta} d \psi=\lim _{n \rightarrow \infty}\left(C_{i-1, n}+C_{i-2, n}+\cdots+C_{i-m, n}\right) .
\end{aligned}
$$

It follows that the values of the integrals $\int_{\xi^{\prime \prime}+\delta}^{\xi^{\prime}-\delta} d \psi(=0), \int_{\xi^{\prime}-\delta}^{\xi^{\prime}+\delta} d \psi, \int_{\xi^{\prime \prime}-\delta}^{\xi^{\prime \prime}+\delta} d \psi$ do not depend on $\delta$. This means that $\psi(x)$ is constant in the intervals $\left(\xi^{\prime \prime}, \xi^{\prime}\right)$, $\left(\xi^{\prime}, \xi^{\prime}+\delta\right),\left(\xi^{\prime \prime}-\delta, \xi^{\prime \prime}\right)$, so that

$$
\int_{\xi^{\prime}-\delta}^{\xi^{\prime}+\delta} d \psi=\psi\left(\xi^{\prime}+0\right)-\psi\left(\xi^{\prime}-0\right), \quad \int_{\xi^{\prime \prime}-\delta}^{\xi^{\prime \prime}+\delta} d \psi=\psi\left(\xi^{\prime \prime}+0\right)-\psi\left(\xi^{\prime \prime}-0\right) .
$$

Hence, by virtue of the " $C$-property," $m=1, l=0$. Furthermore, the assumption $\psi\left(\xi^{\prime}+\delta\right)-\psi\left(\xi^{\prime}-\delta\right)=0$ implies $\psi(x)=$ const. in $\left(\xi^{\prime \prime}, \xi^{\prime}\right)$, which requires that $c_{i-1, n}$ be to the left of $\xi^{\prime \prime}$, with a similar conclusion from the assumption $\psi\left(\xi^{\prime \prime}+\delta\right)-\psi\left(\xi^{\prime \prime}-\delta\right)=0$. Finally, we notice that we cannot have simultaneously

$$
\psi\left(\xi^{\prime \prime}+\delta\right)-\psi\left(\xi^{\prime \prime}-\delta\right)=0, \quad \psi\left(\xi^{\prime}+\delta\right)-\psi\left(\xi^{\prime}-\delta\right)=0,
$$

for then $\psi(x)=$ const. in $\left(\xi^{\prime \prime}-\delta, \xi^{\prime}+\delta\right)$, and this, by the "C -property," contradicts (80). Thus, if (80) is satisfied, then: $(\alpha) l=0, m=1 ;(\beta) \psi(x)$ is constant in $\left(\xi^{\prime \prime}, \xi^{\prime}\right),\left(\xi^{\prime}, \xi^{\prime}+\delta\right),\left(\xi^{\prime \prime}-\delta, \xi^{\prime \prime}\right) ;(\gamma) \lim _{n \rightarrow \infty} C_{i, n}=\psi\left(\xi^{\prime}+0\right)-\psi\left(\xi^{\prime}-0\right)$ $=\sigma_{1}, \lim _{n \rightarrow \infty} C_{i-1, n}=\psi\left(\xi^{\prime \prime}+0\right)-\psi\left(\xi^{\prime \prime}-0\right)=\sigma_{2} ;(\delta){\sigma_{1}}^{2}+\sigma_{2}{ }^{2} \neq 0$, i.e., one at least of the points $\xi^{\prime}, \xi^{\prime \prime}$ is a point of discontinuity of $\psi(x)$ and the saltus is the limiting value of the corresponding coefficient. Moreover, if, for instance, $\psi(x)$ is continuous at $x=\xi^{\prime}$, then $c_{i-1, n} \leqq \xi^{\prime \prime}(n \geqq N) ;(\epsilon) c_{i, n}$ and $c_{i-1, n}$ cannot

* All limits $\xi^{\prime}, \xi^{\prime \prime}, \cdots$ here considered are assumed to belong to $[a, b]$. If $\xi^{\prime}=b$ or $\xi^{\prime \prime}=a$, then $\xi^{\prime}+\delta, \xi^{\prime \prime}-\delta$ should be replaced by $b$ or $a$ respectively. Here and hereafter $\delta$ or $N$ denote properly chosen sufficiently small or sufficiently large numbers respectively, which may be different in different formulae. 
both belong to the interval $\left(\xi^{\prime \prime}, \xi^{\prime}\right)(n \geqq N)$. It follows that if one at least of the conditions $l>0$ or $m>1$ is satisfied, then the third condition (80.3) cannot hold.

By the foregoing, any subinterval $(c, d) \subset(a, b)$ which has no abscissas $c_{i, n}$, for $n \geqq N$, is an interval of constancy for $\psi(x)$. More precisely, the zeros of $\omega_{n}(x)$ are everywhere dense in any subinterval $\left[a_{1}, b_{1}\right]$ which is not an interval of constancy for $\psi(x)$, i.e., $\left[a_{1}, b_{1}\right]$ contains at least one zero of $\omega_{n}(x)$, for $n \geqq N$, in its interior (or at its end-points, if $\psi(x)$ is continuous at $x=a_{1}, b_{1}$ ). (We omit the proof, since it is quite similar to that of Fejér, loc. cit., pp. 308309.)

This property can be generalized as follows: Let a subinterval $[c, d]$ contain no more than one abscissa $c_{i_{\nu}, n_{\nu}}$ for $n=n_{1}, n_{2}, \cdots, n_{\nu}, \cdots *$ Then, if $\psi(x)$ is continuous at $x=c_{1}, d_{1}\left(c \leqq c_{1}<d_{1} \leqq d\right)$

$$
\lim _{\nu \rightarrow \infty} C_{i \nu, n_{\nu}}=\int_{c_{1}}^{d_{1}} d \psi(x) .
$$

Hence, $\psi(x)=$ const. in $(c, d) ; \lim _{\nu \rightarrow \infty} C_{i_{\nu}, n_{\nu}}=0$.

The above results hold for any convergent $F M Q$ formula. Suppose now that all abscissas lie in $[a, b]$. We can state some more properties as follows (again derived by reasoning similar to that of Fejér).

In a convergent $F M Q$ formula corresponding to a finite interval either all coefficients $\rightarrow 0$, as $n \rightarrow \infty$, or else, $\psi(x)$ has discontinuities in $(a, b)$. Hence, if $\psi(x)$ is continuous throughout $[a, b]$ (and a fortiori, if $d \psi(x)=p(x) d x, p(x) \geqq 0$ and integrable in $(a, b))$, then $\lim _{n \rightarrow \infty} C_{i, n}=0, i=1,2, \cdots, n$.

We may go still further if we assume $q=2 n-1$, i.e., when dealing with a $G M Q$ formula. Here we have the following result.

If in a GMQ formula

$$
\lim _{n \rightarrow \infty} c_{i, n}=\xi_{i}, \quad i=1,2, \cdots,
$$

then $\psi(x)$ is a step-function with saltus $\sigma_{i}$ at $x=\xi_{i}$, and $\lim _{n \rightarrow \infty} C_{i, n}=\sigma_{i}$, $i=1,2, \cdots$.

The statement concerning $C_{i, n}$ follows as above in $(\gamma)$. As to the behavior of $\psi(x)$, (81) implies, by the preceding, that $\psi(x)$ is constant in the intervals $\left(\xi_{i-1}, \xi_{i}\right)$. Moreover, were $\psi(x)$ continuous at a certain point $x=\xi_{i}, \xi_{i-1}$ and

* This is possible. Consider, for example, $\omega_{n}(x)=\Phi_{n}(x ; d \psi)$, where $(a, b) \equiv(-h, h), d \psi=p(x) d x$, $p(-x) \equiv p(x), p(x)=0$ in $\left(-h_{1}, h_{1}\right) \subset(-h, h)$. Then $c_{n+1,2 n+1}=0, n=0,1, \cdots$, and this is the only zero of $\omega_{2 n+1}(x)$ in $\left[-h_{1}, h_{1}\right]$, while $\omega_{2 n}(x)$ has no zeros in this interval. 
$\xi_{i+1}$ would have been points of discontinuity, so that $\psi(x)=$ const. in the interval $\left(\xi_{i-1}, \xi_{i+1}\right)$, which could contain one abscissa only, namely, $c_{i, n}$, and

$$
c_{i-1, n} \leqq \xi_{i-1}, c_{i-2, n} \leqq \xi_{i-2}, \cdots ; c_{i+1, n} \geqq \xi_{i+1}, c_{i+2, n} \geqq \xi_{i+2}, \cdots .
$$

This contradicts $(81)$, for all $c_{i, n}$ lie in $(a, b)$, while $c_{1, n}$ decreases and $c_{n, n}$ increases, as $n$ increases. It follows that if $\psi(x)$ is continuous throughout $[a, b]$, with no subintervals of constancy, then neither (80) nor (81) is possible.

The above results generalize those known for $G M Q$ formulae. It is interesting to note that in their proof there was no need to invoke the theory of algebraic continued fractions nor that of the Moment-Problem (on the contrary, the preceding results are applicable to the latter problem). We have made use of but a small part of our convergence theorems, namely, of the fact that the $F M Q$ formula under discussion converges for any $f(x)$ which is constant in any finite subinterval, arbitrarily chosen, and vanishes elsewhere. Thus, our results hold for an infinite interval as well, once the above convergence property is established for such functions.

13. The sequence $\left\{\omega_{n}(x)\right\}$ forms an $O P$ sequence. We close with a brief discussion of the following interesting question.

Under what conditions is the sequence

$$
\begin{aligned}
\omega_{n}(x)= & \Phi_{n}(x)+A_{1} \Phi_{n-1}(x)+\cdots+A_{k-1} \Phi_{n-k+1}(x) \\
& \left(n=0,1, \cdots ; \Phi_{-i}(x) \equiv 0, A_{1}, A_{2}, \cdots, A_{k-1}=\text { const. }\right)
\end{aligned}
$$

itself a sequence of $O P$ ?

The solution offered below, although incomplete, is of interest, for it introduces a rather unusual kind of $O P$, where the corresponding $\psi(x)$ has a subinterval of constancy. It also offers a good illustration of the preceding results. In what follows we discuss - and incompletely-the following case only:

$$
\begin{gathered}
\omega_{n}(x)=\Phi_{n}(x)+A \Phi_{n-1}(x) \\
\left(n=0,1, \cdots ; \Phi_{-1}(x) \equiv 0 ; A \neq 0, \text { independent of } n\right) .
\end{gathered}
$$

The answer to the proposed question will be in the affirmative if and only if a recurrence relation of the form (5) exists for the $\left\{\omega_{n}(x)\right\}$ :

$$
\begin{array}{r}
\omega_{n+2}(x) \equiv \omega_{n+2}=\left(x-c_{n+2}^{\prime}\right) \omega_{n+1}(x)-\lambda_{n+2}^{\prime} \omega_{n}(x) \\
\left(n \geqq 0, \omega_{0}=1, \omega_{1}=x-c_{1}^{\prime}\right),
\end{array}
$$

where $\lambda_{1}{ }^{\prime}(>0)$ and $c_{1}^{\prime}$ are arbitrary, and all $\lambda_{n}{ }^{\prime}$ are positive. Substituting here for $\omega_{n}, \omega_{n+1}, \omega_{n+2}$ their expressions (82) and making use of (5), we get: 


$$
\begin{aligned}
c_{n+2}^{\prime}-c_{n+2} & =0, \lambda_{n+2}^{\prime}-\lambda_{n+2}+A\left(c_{n+2}^{\prime}-c_{n+1}\right)=0, n=0,1, \cdots, \\
\lambda_{n+2}^{\prime}-\lambda_{n+1} & =0, \quad n=1,2, \cdots,
\end{aligned}
$$

whence,

$$
\begin{aligned}
c_{1}^{\prime} & =c_{1}-A ; \quad c_{n}^{\prime}=c_{n}, \quad n=2,3, \cdots, \\
\lambda_{n}^{\prime} & =\lambda_{n-1}, \quad n=3,4, \cdots ; \quad \lambda_{2}^{\prime}=\lambda_{2}-A\left(c_{2}-c_{1}\right),
\end{aligned}
$$

so that the given sequence of $O P,\left\{\Phi_{n}(x)\right\}$, must satisfy the following conditions:

$$
\lambda_{n+1}-\lambda_{n+2}+A\left(c_{n+2}-c_{n+1}\right)=0, \quad n=1,2, \cdots .
$$

Here we try to satisfy (85) in the following special manner:

$$
c_{n+2}-c_{n+1}=h-\text { const., independent of } n \quad(n=1,2, \cdots) .
$$

The above relations now yield

$$
\begin{aligned}
& \lambda_{n+1}-\lambda_{n+2}=-A h, \quad n=1,2, \cdots, \\
& c_{n}=(n-2) h+c_{2}, \quad \lambda_{n}=(n-2) A h+\lambda_{2}, \quad n=3,4, \cdots,
\end{aligned}
$$

$\lambda_{1}, \lambda_{2}>0$ and $c_{1}, c_{2}$ arbitrary; $A h \geqq 0$. Taking (without loss of generality) $\lambda_{1}{ }^{\prime}=\lambda_{1}=1$ and changing notations, we state the following result.

Consider the continued fractions

$$
\begin{gathered}
F(x) \equiv \frac{1 \mid}{\mid x-c}-\frac{\lambda \mid}{\mid x}-\frac{\lambda+A h \mid}{\mid x-h}-\cdots-\frac{\lambda+n A h \mid}{\mid x-n h}-\cdots, \\
F_{1}(x) \equiv \frac{1 \mid}{\mid x-c+A}-\frac{\lambda+A c \mid}{\mid x}-\frac{\lambda \mid}{\mid x-h}-\frac{\lambda+A h \mid}{\mid x-2 h}-\cdots \\
-\frac{\lambda+(n-1) A h \mid}{\mid x-n h}-\cdots,
\end{gathered}
$$

where the constants $\lambda, A, c$, h are such that

$$
\lambda>0, \quad \lambda+A c>0, \quad A h \geqq 0 .
$$

The denominators of the successive convergents to (89) and (90), which we denote respectively by $\left\{\Phi_{n}(x)\right\},\left\{\omega_{n}(x)\right\}$ give a solution of our problem, i.e., each sequence $\left\{\Phi_{n}(x)\right\},\left\{\omega_{n}(x)\right\}$ is an OP sequence (which is not new), and their mutual relation is expressed in (82).

What can be said of the orthogonality intervals and of the corresponding $\psi(x), \psi_{1}(x)$ for each sequence?

Leaving aside the case $A h>0$ which leads to infinite intervals of orthogonality, assume $h=0$. We find from (89), (90): 


$$
\begin{aligned}
& \text { (92) } \quad F(x)=2 /\left\{x-2 c+\left(x^{2}-4 \lambda\right)^{1 / 2}\right\} \\
& \text { (93) } \quad F_{1}(x)=2 \lambda^{2} /\left\{2 \lambda^{2}(x-A-c)-(\lambda+A c)\left(x+\left(x^{2}-4 \lambda\right)^{1 / 2}\right)\right\} .
\end{aligned}
$$

For the sake of brevity, we make the additional assumption $c=0$. Making use of some results of Stieltjes, ${ }^{*}$ we state our final results as follows. For the sequence $\left\{\Phi_{n}(x)\right\}$ :

$$
\begin{aligned}
F(x) & =\frac{1 \mid}{\mid x}-\frac{\lambda \mid}{\mid x}-\frac{\lambda \mid}{\mid x}-\cdots=\left\{x-\left(x^{2}-4 \lambda\right)^{1 / 2}\right\} / 2 \lambda \\
& =\frac{1}{2 \pi \lambda} \int_{-2 \lambda^{1 / 2}}^{2 \lambda^{1 / 2}}\left(4 \lambda-y^{2}\right)^{1 / 2} \frac{d y}{x-y},
\end{aligned}
$$

$\left(\lambda>0 ; x\right.$ real or complex, not on $\left.\left[-2 \lambda^{1 / 2}, 2 \lambda^{1 / 2}\right]\right)$.

The interval of orthogonality is $\left(-2 \lambda^{1 / 2}, 2 \lambda^{1 / 2}\right)$ and

$$
\psi(x)=\frac{1}{2 \pi \lambda} \int_{-2 \lambda^{1 / 2}}^{x}\left(4 \lambda-y^{2}\right)^{1 / 2} d y .
$$

For the sequence $\left\{\omega_{n}(x)\right\}$ :

$$
\begin{aligned}
F_{1}(x) & =\frac{1-\lambda / A^{2}}{x-\alpha}+\frac{1}{A} \int_{-2 \lambda^{1 / 2}}^{2 \lambda^{1 / 2}} \frac{\left(4 \lambda-y^{2}\right)^{1 / 2}}{y-\alpha} \frac{d y}{x-y} \\
& =\left\{x+2 A+\left(x^{2}-4 \lambda\right)^{1 / 2}\right\} / 2 A(x-\alpha), \quad \alpha=-A-\lambda / A .
\end{aligned}
$$

In case $A>0$ the interval of orthogonality is $\left(\alpha, 2 \lambda^{1 / 2}\right)$, and

$$
\begin{aligned}
& \psi_{1}(\alpha)=0, \psi_{1}(x)=\text { const. }=1-\lambda / A^{2} \text { on }\left(\alpha,-2 \lambda^{1 / 2}\right] \\
& \psi_{1}(x)=1-\frac{\lambda}{A^{2}}+\frac{1}{A} \int_{-2 \lambda^{1 / 2}}^{x}\left(4 \lambda-y^{2}\right)^{1 / 2} \frac{d y}{y-\alpha} \text { on }\left[-2 \lambda^{1 / 2}, 2 \lambda^{1 / 2}\right] .
\end{aligned}
$$

In case $A<0$ the interval of orthogonality is $\left(-2 \lambda^{1 / 2}, \alpha\right)$, and

$$
\begin{aligned}
& \psi_{1}(x)=-\frac{1}{A} \int_{-2 \lambda^{1 / 2}}^{x} \frac{d \psi(y)}{\alpha-y} \text { on }\left[-2 \lambda^{1 / 2}, 2 \lambda^{1 / 2}\right] \\
& \psi_{1}(x)=\text { const. }=-\frac{1}{A} \int_{-2 \lambda^{1 / 2}}^{2 \lambda^{1 / 2}} \frac{d \psi(y)}{\alpha-y}
\end{aligned}
$$

on $\left[2 \lambda^{1 / 2}, \alpha\right)$,

$$
\psi_{1}(\alpha)=-\frac{1}{A} \int_{-2 \lambda^{1 / 2}}^{2 \lambda^{1 / 2}} \frac{d \psi(y)}{\alpha-y}+1-\frac{\lambda}{A^{2}} .
$$

Thus, in both cases

* Stieltjes, Recherches sur les fractions continues, Oeuvres, vol. II, pp. 402-566; pp. 509-510. 


$$
d \psi_{1}(x)=\frac{1}{A} \frac{d \psi(x)}{x-\alpha} \text { in }\left(-2 \lambda^{1 / 2}, 2 \lambda^{1 / 2}\right) .
$$

Note that all zeros $c_{i, n}$ of $\omega_{n}(x)$ lie in $\left(-2 \lambda^{1 / 2}, 2 \lambda^{1 / 2}\right)$, except one. Moreover (in accordance with the general theory and with the preceding discussion), as $n \rightarrow \infty$,

$$
\begin{aligned}
& c_{1, n} \rightarrow \alpha, c_{n, n} \rightarrow 2 \lambda^{1 / 2}, \text { if } A>0, \\
& c_{1, n} \rightarrow-2 \lambda^{1 / 2}, c_{n, n} \rightarrow \alpha, \text { if } A<0,
\end{aligned}
$$

where $\alpha$, introduced above, is the zero of the polynomial

$$
H(w) \equiv w+A \equiv \frac{x+\left(x^{2}-4 \lambda\right)^{1 / 2}}{2}+A \quad(|w| \rightarrow \infty, \text { as }|x| \rightarrow \infty) .
$$

$\psi_{1}(x)$ has no saltus if and only if $A^{2}=\lambda$; then the two intervals of orthogonality obtained above coincide with $\left(-2 \lambda^{1 / 2}, 2 \lambda^{1 / 2}\right)$, and

$$
d \psi_{1}(x)=\frac{1}{\lambda^{1 / 2}} \frac{d \psi(x)}{2 \lambda^{1 / 2}-x} .
$$

Untversity of Pennsylvanta,

Philadelphia, Pa. 